\title{
DETECTION OF THE SPLASHBACK RADIUS AND HALO ASSEMBLY BIAS OF MASSIVE GALAXY
} CLUSTERS

\author{
Surhud More ${ }^{1}$, Hironao Miyatake ${ }^{1,2,3}$, Masahiro Takada ${ }^{1}$, Benedikt Diemer ${ }^{4}$, Andrey V. Kravtsov ${ }^{5,6,7}$, \\ Neal K. Dalal ${ }^{1,8}$, Anupreeta More ${ }^{1}$, Ryoma Murata ${ }^{1,9}$, Rachel Mandelbaum ${ }^{10}$, Eduardo Rozo ${ }^{11}$, Eli S. Rykoff ${ }^{12}$, \\ Masamune Oguri ${ }^{1,9,13}$, AND David N. SPergel ${ }^{1,3}$ \\ ${ }^{1}$ Kavli Institute for the Physics and Mathematics of the Universe (WPI), Tokyo Institutes for Advanced Study, The University of Tokyo, 5-1-5 Kashiwanoha, \\ Kashiwa-shi, Chiba, 277-8583, Japan; surhud.more@ipmu.jp \\ 2 Jet Propulsion Laboratory, California Institute of Technology, Pasadena, CA 91109, USA \\ ${ }^{3}$ Department of Astrophysical Sciences, Princeton University, Peyton Hall, Princeton, NJ 08544, USA \\ ${ }^{4}$ Harvard-Smithsonian Center for Astrophysics, 60 Garden St., Cambridge, MA 02138, USA \\ ${ }^{5}$ Department of Astronomy and Astrophysics, The University of Chicago, Chicago, IL 60637, USA \\ ${ }^{6}$ Kavli Institute for Cosmological Physics, The University of Chicago, Chicago, IL 60637, USA \\ ${ }^{7}$ Enrico Fermi Institute, The University of Chicago, Chicago, IL 60637, USA \\ ${ }^{8}$ Department of Physics, University of Illinois Urbana-Champagne, 1110 West Green Street Urbana, IL 61801-3080, USA \\ ${ }^{9}$ Department of Physics, University of Tokyo, 7-3-1 Hongo, Bunkyo-ku, Tokyo 113-0033, Japan \\ ${ }^{10}$ McWilliams Center for Cosmology, Department of Physics, Carnegie Mellon University, Pittsburgh, PA 15213, USA \\ ${ }^{11}$ Department of Physics, University of Arizona, 1118 E 4th St, Tucson, AZ 85721, USA \\ ${ }^{12}$ SLAC National Accelerator Laboratory, Menlo Park, CA 94025, USA \\ ${ }^{13}$ Research Center for the Early Universe, University of Tokyo, 7-3-1 Hongo, Bunkyo-ku, Tokyo 113-0033, Japan \\ Received 2016 January 25; revised 2016 April 18; accepted 2016 May 1; published 2016 June 27
}

\begin{abstract}
We show that the projected number density profiles of Sloan Digital Sky Survey photometric galaxies around galaxy clusters display strong evidence for the splashback radius, a sharp halo edge corresponding to the location of the first orbital apocenter of satellite galaxies after their infall. We split the clusters into two subsamples with different mean projected radial distances of their members, $\left\langle R_{\mathrm{mem}}\right\rangle$, at fixed richness and redshift. The sample with smaller $\left\langle R_{\text {mem }}\right\rangle$ has a smaller ratio of the splashback radius to the traditional halo boundary $R_{200 m}$ than the subsample with larger $\left\langle R_{\mathrm{mem}}\right\rangle$, indicative of different mass accretion rates for these subsamples. The same subsamples were recently used by Miyatake et al. to show that their large-scale clustering differs despite their similar weak lensing masses, demonstrating strong evidence for halo assembly bias. We expand on this result by presenting a $6.6 \sigma$ difference in the clustering amplitudes of these samples using cluster-photometric galaxy crosscorrelations. This measurement is a clear indication that halo clustering depends on parameters other than halo mass. If $\left\langle R_{\text {mem }}\right\rangle$ is related to the mass assembly history of halos, the measurement is a manifestation of the halo assembly bias. However, our measured splashback radii are smaller, while the strength of the assembly bias signal is stronger, than the predictions of collisionless $\Lambda$ cold dark matter simulations. We show that dynamical friction, cluster mis-centering, or projection effects are not likely to be the sole source of these discrepancies. However, further investigations regarding unknown catastrophic weak lensing or cluster identification systematics are warranted.
\end{abstract}

Key words: dark matter - cosmology: observations - galaxies: clusters: general - large-scale structure of universe methods: observational

\section{INTRODUCTION}

Dark matter halos with masses larger than $10^{14} h^{-1} M_{\odot}$ collapse out of dense peaks in the primordial Gaussian density fluctuations that are believed to originate from quantum fluctuations in cosmic inflation (see e.g., Kaiser 1984; Bardeen et al. 1986; see Kravtsov \& Borgani 2012 for a recent review). Clusters of galaxies form within such massive dark matter halos. The large-scale clustering amplitudes of the halos hosting galaxy clusters are thus heavily biased compared to the underlying matter distribution (Kaiser 1984; Mo \& White 1996; Sheth et al. 2001; Tinker et al. 2010).

Although the large-scale clustering amplitude of dark matter halos primarily depends on halo mass, it also depends on other secondary parameters correlated with halo assembly history (Gao et al. 2005; Wechsler et al. 2006; Gao \& White 2007). For cluster-sized halos, the dependence of the clustering amplitude on these secondary parameters can be traced back to the properties of the primordial density peaks from which such halos form, for example, the radial profile of the initial peaks (Dalal et al. 2008). The dependence of the large-scale clustering amplitude on various parameters other than the halo mass has been broadly referred to as halo assembly bias, and has been studied in great detail using cosmological simulations (Sheth \& Tormen 2004; Gao et al. 2005; Wechsler et al. 2006; Gao \& White 2007; Li et al. 2008).

Halo assembly bias has, however, been difficult to establish in astrophysical observations. A clean detection of halo assembly bias requires identifying samples of isolated halos which are matched in their halo masses but differ in their assembly histories. There have been several claims of detection of halo assembly bias on galaxy scales in the literature (e.g., Yang et al. 2006; Tinker et al. 2012; Hearin et al. 2015). However, Lin et al. (2016) investigated the first of these claims and found no strong evidence for halo assembly bias on galaxy scales. The difference in the conclusions was a result of contamination of the halo samples by satellite galaxies, or the differences in halo masses of the samples used to look for halo assembly bias (Lin et al. 2016). 
Recently, Miyatake et al. (2016) presented the first evidence of halo assembly bias on cluster scales. Galaxy clusters offer two advantages: first, the probability of a cluster-sized halo being a satellite of an even bigger halo is much smaller than in the case of galaxies, and second, the weak gravitational lensing signal can be used to match galaxy cluster subsamples for their halo masses with a greater accuracy.

The galaxy cluster subsamples used by Miyatake et al. (2016) were drawn from the Sloan Digital Sky Survey (SDSS) red-sequence Matched-filter Probabilistic Percolation (redMaPPer) galaxy cluster catalog, and were matched in redshift and richness distribution, but differed in the compactness of the member galaxy distribution. These samples were shown to have very similar masses based on weak lensing, but had different large-scale biases. The main goal of this paper is to observationally establish the connection between the member galaxy distribution and the mass assembly of these cluster subsamples without relying on proxies related to complicated baryonic physics, such as star formation rates.

For this purpose, we use a unique probe of the mass assembly of galaxy clusters, which relies on the observational detection of the edges of galaxy clusters. Models of self-similar secondary infall of matter onto a spherical overdensity predict the presence of a density jump at the location where recently accreted material is reaching its first apocenter, associated with the last density caustic (Fillmore \& Goldreich 1984; Bertschinger 1985). Although the collapse of matter onto realistic density peaks in cold dark matter (CDM) models is considerably more complex than that envisioned in these models, the last density caustic manifests itself as a sharp steepening of the density profile in dark matter halos (Diemer \& Kravtsov 2014). This caustic is also called the splashback radius or the second turnaround radius.

Note that the mere detection of the steepest slope of the density profile near the one-halo to two-halo transition does not constitute a detection of the splashback radius. Such a steepest slope radius will always be present, regardless of whether the density distribution around halos exhibits sharp caustics. The steepening associated with the splashback radius in rapidly accreting halos is stronger than that expected from a simple sum of the Einasto profile and a large-scale two-halo term. Around such halos, the three-dimensional distribution of mass has edge-like features associated with the caustic shell, which can manifest themselves as a region of rapid steepening of the slope in the spherically averaged density profile (Adhikari et al. 2014; Diemer \& Kravtsov 2014; More et al. 2015).

The splashback radius can be used to define a physical boundary for dark matter halos (More et al. 2015). The splashback radius crucially depends upon the mass accretion rate of the collapsing halo (Vogelsberger et al. 2011; Adhikari et al. 2014; Diemer \& Kravtsov 2014). For halos of the same mass, a large accretion rate results in a smaller splashback radius. The physical reason for this is simple: the deeper the halo potential well becomes during the orbit of a dark matter particle, the smaller is the value of its apocenter.

As discussed in More et al. (2015), hints for the splashback radius may have been seen before for individual clusters (Rines et al. 2013; Tully 2015). In particular, Patej \& Loeb (2015) considered a sample of 60 galaxy clusters and claimed some statistical evidence for the presence of the steepening associated with the splashback radius in roughly a third of these clusters. However, detecting the splashback radius for individual systems, as done in all the studies mentioned above, is complicated due to the presence of substructure in the outskirts as well as the triaxiality of clusters. In this paper, we will harness the power of statistics to present the first high signal-to-noise detection of the splashback radius for our galaxy cluster subsamples relying on stacking methods. We will use the splashback radius to establish that these galaxy cluster subsamples have different mass accretion rates and have different large-scale clustering amplitudes, a signature of halo assembly bias.

This paper is organized as follows:

1. Section 2 describes the cluster subsamples and the SDSS photometric galaxy data which form the basis of our study, and the methods we adopt in order to obtain the measurements of the galaxy number densities around our cluster subsamples.

2. Section 3 presents our measurements of the galaxy number densities around our cluster subsamples, our inferences for the location of the splashback radius from these measurements, and our detection of halo assembly bias.

3. Section 4 presents the predictions for the location of the splashback radius and the amount of halo assembly bias from numerical simulations in the context of the standard cosmological model. In particular, we discuss a number of systematic effects which could affect our interpretation.

4. The broad implications of our results are discussed in Section 5, and conclusions and a summary are presented in Section 6.

Throughout this paper, we adopt a flat $\Lambda \mathrm{CDM}$ cosmological model with matter density parameter $\Omega_{\mathrm{m}}=0.27$ and the Hubble parameter $h=0.7$, unless otherwise stated. We use log to denote logarithms with respect to base 10 . We will use $r$ to denote threedimensional distances, and $R$ for projected distances between galaxies or between galaxies and cluster centers. For cases where we want to preserve the notations from previous papers, such as using $R_{\mathrm{sp}}$ for the splashback radius, we will specifically mention whether it is two-dimensional or three-dimensional to avoid confusion. The subscript $200 \mathrm{~m}$ on halo mass $M$ or radius $R$ will refer to the mass or radius corresponding to spherical overdensity halos such that their boundaries enclose 200 times the mean matter density of the universe.

\section{DATA AND METHODS}

We start from the publicly available catalog of galaxy clusters identified from the SDSS Data Release 8 (DR8) photometric galaxy catalog using the redMaPPer cluster finding algorithm (v5.10, see the website ${ }^{14}$ for details and Rykoff et al. 2014; Rozo et al. 2015). The cluster finder uses the ugriz magnitudes and their errors, to identify overdensities of red-sequence galaxies with similar colors as galaxy clusters. For each cluster, the catalog contains an optical richness estimate $\lambda$, a photometric redshift estimate $z_{\lambda}$, as well as the position and centering probabilities of five candidate central galaxies $p_{\text {cen }}$. A separate member galaxy catalog provides a list of members for each cluster, each of which is assigned a membership probability, $p_{\text {mem }}$.

The parent cluster catalog used in Miyatake et al. (2016) consists of an approximately volume limited sample of 8,648 redMaPPer clusters with $20<\lambda<100$ and $0.1 \leqslant z_{\lambda} \leqslant 0.33$. The average and median redshifts of our subsamples are 0.24 and 0.25 , respectively. Throughout this paper we use the

\footnotetext{
${ }^{14}$ http://risa.stanford.edu/redmapper/
} 
position of the most probable central galaxy in each cluster region as a proxy of the cluster center. However, we will discuss the effect of mis-centering on our conclusions in Section 4.3.

In this paper, we subdivide this galaxy cluster sample into two subsamples following the same procedure as in Miyatake et al. (2016). Briefly, we obtain the average projected clustercentric separation of member galaxies, $\left\langle R_{\text {mem }}\right\rangle$, for each cluster, and compute the median $\left\langle R_{\mathrm{mem}}\right\rangle$ as a function of richness and redshift. ${ }^{15}$ We use this median to divide the parent sample into two subsamples. The large- and small- $\left\langle R_{\text {mem }}\right\rangle$ subsamples, labeled as low- and high- $c_{\mathrm{gal}}$ in this paper, consist of 4235 and 4413 clusters, respectively.

In order to compute galaxy surface number density around these cluster subsamples, we make use of the photometric galaxy catalog from SDSS DR8 (Aihara et al. 2011). We exclude galaxies with any of the following flags: SATURATED, SATUR_CENTER, BRIGHT, DEBLENDED_AS_MOVING. We correct the magnitudes for galactic dust extinction using the maps of Schlegel et al. (1998), and use all photometric galaxies with extinction corrected $i$-band model magnitudes brighter than 21.0 and with magnitude errors less than 0.1 .

We compute the stacked surface number densities of the SDSS photometric galaxy samples around each of our cluster subsamples as a function of comoving projected separation, $R$, from the galaxy cluster center. Since our cluster subsamples span a wide range in redshift $(0.1 \leqslant z \leqslant 0.33)$, the surface density profiles around lower redshift clusters will systematically contribute galaxies from a fainter photometric galaxy population. To avoid such biases, for our fiducial analysis we only count cluster-galaxy pairs if the photometric galaxy has an absolute magnitude of $M_{i}-5 \log h<-19.43^{16}$, assuming that it is located at the redshift of the cluster (this limit corresponds to an apparent magnitude of $m_{i}=21$ at $z=0.33$ for our assumed cosmological model). Additionally, we will also present results for photometric galaxies that are one and two magnitudes brighter than our fiducial measurement, to explore the dependence of the splashback radius on the magnitude of the photometric galaxies used.

We expect that the surface density measurement will consist of galaxies correlated with the galaxy clusters under consideration as well as uncorrelated galaxies in the foreground and the background. To determine this uncorrelated component, we compute the galaxy surface density around a sample of random points. We use a 100 times larger number of random points than the number of clusters in our subsamples. ${ }^{17}$ These random points incorporate the survey geometry, depth variations, and distributions of clusters in redshift and richness. We subtract the background around random points from the total to obtain the surface density of correlated galaxies, $\Sigma_{\mathrm{g}}(R) .{ }^{18}$ We use 102

\footnotetext{
15 When computing the average, we weight each galaxy's cluster-centric distance with its membership probability $\left(p_{\text {mem }}\right)$.

16 Note that we do not use any $k$-corrections or corrections for luminosity evolution here, since the redshifts of the photometric galaxies are quite uncertain.

17 We have tested that the use of the improved random catalog from Rykoff et al. (2016) does not change any conclusions in this paper.

18 Note that this measure of the galaxy surface density will underestimate the true surface density by the fraction of area lost due to masks and the survey geometry. We have checked that for scales less than $5 h^{-1} \mathrm{Mpc}$, relevant for splashback radius measurements, the masked area is not significantly dependent on the radius as to affect our results. For the assembly bias measurements, we consider the ratio of the surface densities around the two cluster subsamples, which are statistically affected by the mask in a similar manner, and hence this dependence cancels out when we take a ratio.
}

jackknife regions in order to compute the covariance in the measurements of $\Sigma_{\mathrm{g}}(R)$ with a typical size of $10 \times 10$ sq. deg. which corresponds to about $100 \times 100\left(h^{-1} \mathrm{Mpc}\right)^{2}$ at the median redshift of our cluster subsamples. The jackknife regions are thus significantly larger compared to the scales of interest in this paper, justifying the assumptions behind the jackknife errors.

Using the measurement of the galaxy surface densities, we would like to infer the location of the splashback radius of our cluster subsamples, i.e., the steepest logarithmic slope of the galaxy number density distributions in three dimensions, and establish that this steepening is larger than that expected from a simple sum of Einasto profile and a large-scale two-halo term. Given that the splashback radius is expected to be of the order of $R_{200 \mathrm{~m}}$ of our halos, we fit the surface densities in the range $[0.1,5.0] h^{-1} \mathrm{Mpc}$. The location of the steepening in three dimensions is expected to be different from that in projection (Diemer \& Kravtsov 2014). Therefore, we will use a threedimensional parameterization first proposed by Diemer \& Kravtsov (2014) to forward model the projected measurements (see also More et al. 2015). The model consists of inner and outer surface density profiles with a smooth transition between the two,

$$
\begin{aligned}
\rho_{\mathrm{g}}(r) & =\rho_{\mathrm{g}}^{\text {inner }} f_{\text {trans }}+\rho_{\mathrm{g}}^{\text {outer }} \\
\rho_{\mathrm{g}}^{\text {inner }} & =\rho_{\mathrm{s}} \exp \left(-\frac{2}{\alpha}\left[\left(\frac{r}{r_{\mathrm{s}}}\right)^{\alpha}-1\right]\right), \\
\rho_{\mathrm{g}}^{\text {outer }} & =\rho_{\mathrm{o}}\left(\frac{r}{r_{\text {out }}}\right)^{-s_{\mathrm{e}}}, \\
f_{\text {trans }} & =\left[1+\left(r / r_{\mathrm{t}}\right)^{\beta}\right]^{-\gamma / \beta}, \\
\Sigma_{\mathrm{g}}(R) & =2 \int_{0}^{z_{\max }} \rho_{\mathrm{g}}\left(\sqrt{R^{2}+z^{2}}\right) d z
\end{aligned}
$$

Note that the above fitting formula, which is an Einasto profile in the inner regions which sharply transitions to a power law in the outer regions, is able to reproduce the dark matter profile around halos, and is flexible enough to reproduce the simulation results compared to other fitting formulae such as the Navarro-Frenk-White model and the halo model (Navarro et al. 1996; Oguri \& Hamana 2011; Hikage et al. 2013). Here we simply assume that the same fitting formula is also flexible enough to reproduce the galaxy surface density measured from the SDSS data. ${ }^{19}$ We chose the maximum projection length $z_{\max }=40 h^{-1} \mathrm{Mpc}$ as our default value, and we have checked that the location of the splashback radius is insensitive to this choice, in particular reducing $z_{\max }$ to be as small as $10 h^{-1}$ Mpc.

Given that the parameters $r_{\text {out }}$ and $\rho_{\mathrm{o}}$ are entirely degenerate with each other, we fix $r_{\text {out }}=1.5 h^{-1} \mathrm{Mpc}$. We find that allowing $\alpha$ to vary freely results in an almost perfect degeneracy between $\rho_{\mathrm{s}}$ and $r_{\mathrm{s}}$, with very little impact on the location of the steepening of the galaxy density profiles. Therefore we use a prior on $\log \alpha=\log 0.2 \pm 0.6$, centered at the value expected for the dark matter halos corresponding to our mass estimates from weak lensing (Gao et al. 2008). For our fiducial modeling scheme, we also use Gaussian priors on

\footnotetext{
19 Tests using subhalo density profiles around cluster-scale halos from the simulations presented in Appendix A, as well as the reasonable $\chi^{2}$ values we obtain for describing the observed measurements justify this choice.
} 
$\log \beta=\log 4.0 \pm 0.2$ and $\log \gamma=\log 6.0 \pm 0.2$ centered around the values recommended by Diemer \& Kravtsov (2014) and constrain the parameters $r_{\mathrm{s}}$ and $r_{\mathrm{t}}$ to lie within $[0.1,5.0] h^{-1} \mathrm{Mpc}$.

For our default modeling scheme, we assume that the most probable central galaxy for every cluster (the one with the highest $\left.p_{\text {cen }}\right)$, assigned in the redMaPPer catalog, resides at the true center of gravitational potential in each cluster region. However, as studied in Miyatake et al. (2016) (see also Hikage et al. 2013), some fraction of the central galaxies in our cluster subsamples may be mis-centered, characterized by offset radii ranging from $400 h^{-1} \mathrm{kpc}$ possibly up to $800 h^{-1} \mathrm{kpc}$. If such mis-centered clusters are indeed present in large numbers, our measurements of the splashback radius would be biased high. We will present tests for the effects of mis-centering in Section 4.3.

We will use the affine invariant Markov Chain Monte Carlo (MCMC) sampler of Goodman \& Weare (2010), as implemented in the software package emcee (Foreman-Mackey et al. 2013), in order to sample from the posterior distribution of the parameters, $\log \rho_{\mathrm{s}}, \log r_{\mathrm{s}}, \log \alpha, \log r_{\mathrm{t}}, \log \gamma, \log \beta, \log \rho_{\mathrm{o}}$, and $s_{\mathrm{e}}$, given the galaxy surface density measurements and the stated priors.

As a test of our fitting method, in Appendix A we apply it to projected number density distributions of (sub)halos around galaxy clusters in numerical simulations (see below for the details of the simulations) and show that we are able to recover the location of the steepening of the three-dimensional density distribution of subhalos quite accurately with our modeling scheme.

\section{RESULTS}

\subsection{Splashback in Galaxy Number Density Profiles}

We begin by presenting how the stacked surface density profile of galaxies, $\Sigma_{\mathrm{g}}(R)$, around the entire parent sample of redMaPPer clusters described in the previous section, varies with galaxy samples of different absolute magnitude thresholds. These measurements are shown in the top panels of Figure 1 using points with error bars. The brightness of the photometric galaxy sample increases from left to right. The solid lines in the bottom panels correspond to the profiles of the logarithmic slope of the galaxy surface densities. These slope profiles were obtained using the Savitzky-Golay algorithm to smooth the observed measurements, by fitting a third-order polynomial over a window of five neighboring points, and then using a cubic spline to interpolate between these smoothed measurements. In contrast to the traditional Savitzky-Golay algorithm, we explicitly account for the covariant errors on these data points, as proposed by More (2016). ${ }^{20}$

In Appendix B, we have used subhalo abundance matching to obtain an estimate of the approximate $V_{\text {peak }}$ (the maximum circular velocity of a halo throughout its entire history) value of dark matter subhalos ${ }^{21}$ hosting our photometric galaxies as a function of their magnitude. To compare the observed surface density profiles with those expected from the standard structure formation model, we utilize MultiDark-Planck II (MDPL2), a $3840^{3}$ particle cosmological $N$-body simulation with a box size

\footnotetext{
${ }^{20}$ https://github.com/surhudm/savitzky_golay_with_errors

21 Our use of the term subhalos henceforth includes isolated host halos as well, not just satellite halos. We will use the term halos explicitly when referring to only isolated halos.
}

of $1 h^{-1} \mathrm{Gpc}$ and a mass resolution of $1.51 \times 10^{9} h^{-1} M_{\odot}$ (Klypin et al. 2016). We also use the associated halo catalogs found using Rockstar, a halo finder which groups particles into halos using their phase space information (Behroozi et al. 2013). ${ }^{22}$

The dashed lines in the top and bottom panels of Figure 1 correspond to the expected subhalo surface density profiles around clusters in the cosmological simulation MDPL2 at $z=0.248$. We have selected cluster-sized halos above a mass threshold of $10^{14} h^{-1} M_{\odot}$, which results in the same average halo mass as that of our sample. We have normalized the surface density profiles in the top panels to match the observations at $\sim 11 h^{-1}$ Mpc. There are clear similarities between the density profiles of subhalos in the simulations and the galaxies in observations. The surface number densities strongly deviate from a simple power law and show a clear break on scales of $\sim 1 h^{-1} \mathrm{Mpc}$ in both observations and simulations. This is most clearly seen in the bottom panels, where we see that the profiles reach their steepest slope on scales of $\sim 1 h^{-1} \mathrm{Mpc}$. This steepening, associated with the splashback radius, is also seen in observations, as expected from subhalo surface density profiles in simulations. However, it is also clear that the locations where the surface density profiles reach their steepest slopes are different between observations and simulations, in particular for the left and the middle panels. This discrepancy between the observed and expected splashback radii is also seen for the cluster subsamples, which we investigate at length next. We will extensively quantify, comment on, and explore this discrepancy in the location of splashback radius around these cluster subsamples.

The surface density of photometric galaxies around the lowand high- $c_{\text {gal }}$ cluster subsamples are shown in the upper and lower panels of Figure 2 using orange and purple symbols with error bars, respectively. The lighter shades correspond to photometric galaxies with brighter magnitude limits. The solid lines in the upper and lower right-hand panels of Figure 2 show the logarithmic slope of the surface density profiles around the two subsamples. The slopes for both cluster subsamples reach values steeper than $\sim-1.6$ on either side of $\sim 1 h^{-1} \mathrm{Mpc}$. The surface densities of galaxies around the high- $c_{\text {gal }}$ cluster subsample reach their steepest slopes at a smaller radius compared to the low- $c_{\text {gal }}$ subsample. The value of the steepest slope is considerably larger for the high- $c_{\text {gal }}$ cluster subsample than the low- $c_{\text {gal }}$ subsample. A comparison between the profiles of galaxies as a function of different magnitude thresholds around any given cluster subsample shows very little difference in the location of the steepest slope in projection.

We fit the galaxy surface density profiles with the model described in the previous section. The median and the $68 \%$ confidence intervals of the posteriors of each of these parameters, as well as the best fit $\chi^{2}$ values, are listed in Table 1. The number of degrees of freedom for our model is 8 .

We show the $68 \%$ and $95 \%$ confidence regions from the fits to the surface density of the fiducial sample of photometric galaxies around both our cluster subsamples in the left-hand panel of Figure 3. The corresponding confidence regions for the logarithmic slope, including marginalization over other model parameters, are shown in the right-hand panel. We use the samples from the posterior of the model parameter space to infer the location of the steepest slope of the projected galaxy

$\overline{22}$ These catalogs are publicly available at the website www.cosmosim.org. 

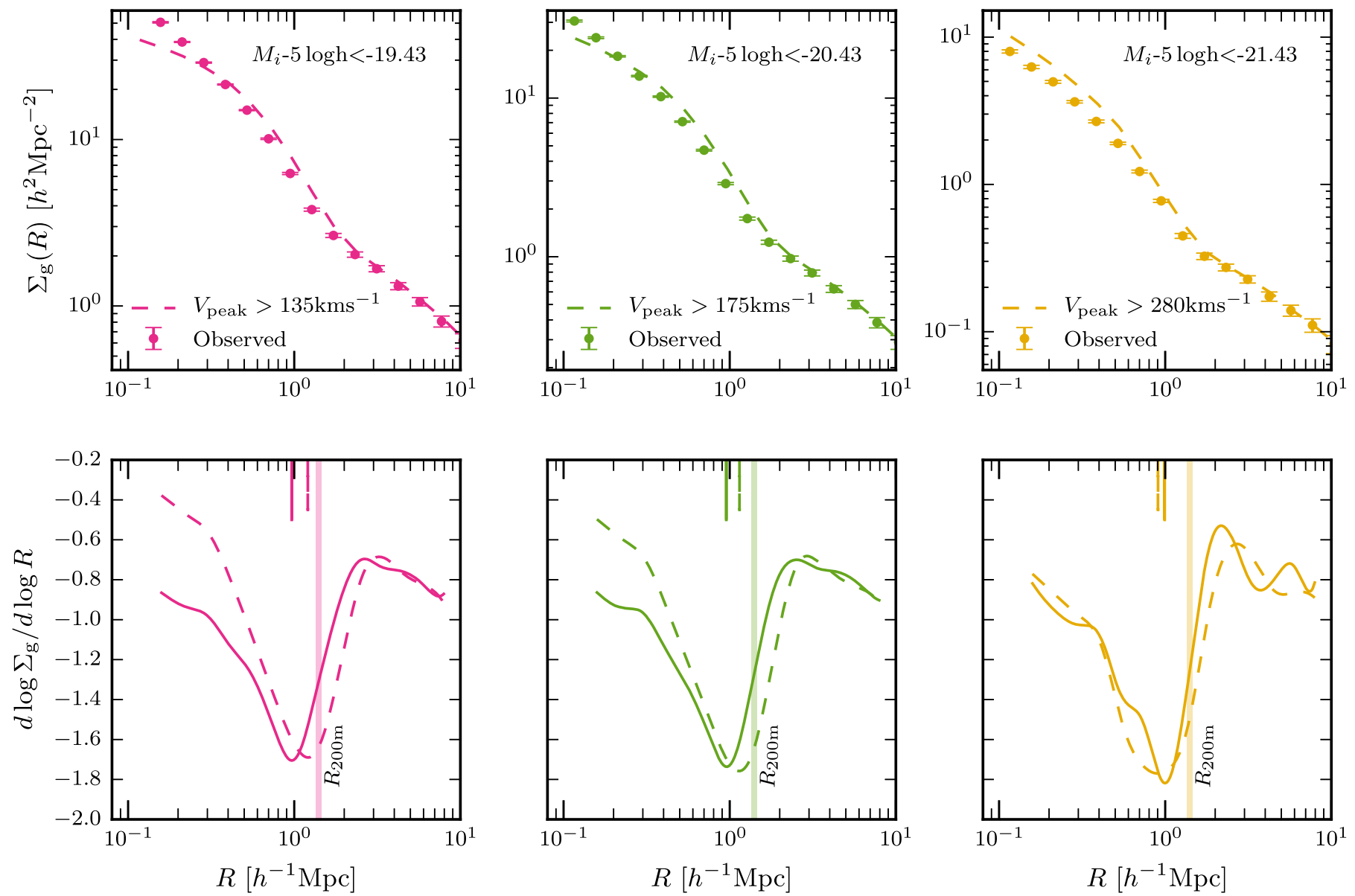

Figure 1. Top panels: the surface number density profiles, $\Sigma_{\mathrm{g}}(R)$, of SDSS photometric galaxies with different magnitude thresholds around the entire redMaPPer cluster sample with $z \in[0.1,0.33]$ and richness $\lambda \in[20,100]$, are shown using symbols with error bars. The dashed lines correspond to (sub)halo surface density profiles in the MDPL2 simulation around clusters with a mass threshold similar to our sample at $z=0.248$. The thresholds on subhalo $V_{\text {peak }}$ values roughly correspond to the magnitude thresholds in each panel and were obtained by subhalo abundance matching (see Appendix B). Bottom panels: the logarithmic slope of the surface density profiles are shown using solid and dashed lines for the observed galaxy and the subhalo surface density profiles, respectively. The observed slope of the surface density profile has a shape which is similar to that expected from simulations. Note that although the surface density profiles both in observations and simulations exhibit similar steepenings, the corresponding radii of the steepest slope are at slightly different locations.

density profile, $R_{\mathrm{sp}}^{2 \mathrm{~d}}$, and its uncertainty. These numbers are reported for all of our subsamples and for the different models in Table 1 as well.

The location of the splashback radius can be compared with the traditional halo boundary definition, $R_{200 \mathrm{~m}}$ for each subsample. This is shown by the vertical shaded bands in the right-hand panels of Figure 2, as estimated from the posterior distribution of the halo masses for our two subsamples inferred from the weak lensing measurement in Miyatake et al. (2016).

We now use the samples from the posterior distribution of model parameters to infer the constraints on the location of the minimum of the logarithmic derivative of the three-dimensional galaxy density profile, $d \log \rho_{\mathrm{g}} / d \log r$. The resultant constraints on $R_{\mathrm{sp}}^{3 \mathrm{~d}}$ are reported in the penultimate column of Table 1 . The inferred value of $R_{\mathrm{sp}}^{3 \mathrm{~d}}$ is always larger than the corresponding $R_{\mathrm{sp}}^{2 \mathrm{~d}}$ for all photometric galaxy samples around both cluster subsamples, as shown explicitly in Figure 4. The vertical dashed line corresponds to the traditional halo boundary definition, $R_{200 \mathrm{~m}}$, for the two subsamples.

Note that, for our model, a transition function $f_{\text {trans }}=1$, would correspond to a simple density profile: a sum of Einasto profile which describes well the inner regions and a power law profile for the outer regions. However, the data strongly disfavor such a model, with $\chi^{2}$ values ranging from 60 to 140 for 9 degrees of freedom depending upon the cluster subsample and the photometric galaxies under consideration. ${ }^{23}$ Therefore, our measurements imply a steepening of the number density profile of galaxies around both of our cluster subsamples beyond that predicted by the sum of an Einasto profile and a power law two-halo term.

\subsection{Detection of Halo Assembly Bias}

The mean number density profile of galaxies correlated with clusters at large separations is proportional to the product of the biases of clusters and galaxies in the photometric sample. We have shown above that these profiles are different for the lowand high- $c_{\text {gal }}$ cluster subsamples. Given that our cluster samples have the same redshift distribution, the bias of photometric galaxies should cancel out in the ratio of the number density profiles, and we can use the ratio to test whether the two cluster

\footnotetext{
23 There is only one additional degree of freedom for these models, as we lose only only one free parameter $r_{\mathrm{t}}$, the other parameters $\gamma$ and $\beta$ have priors in the fiducial modeling scheme and thus do not change the degrees of freedom.
} 

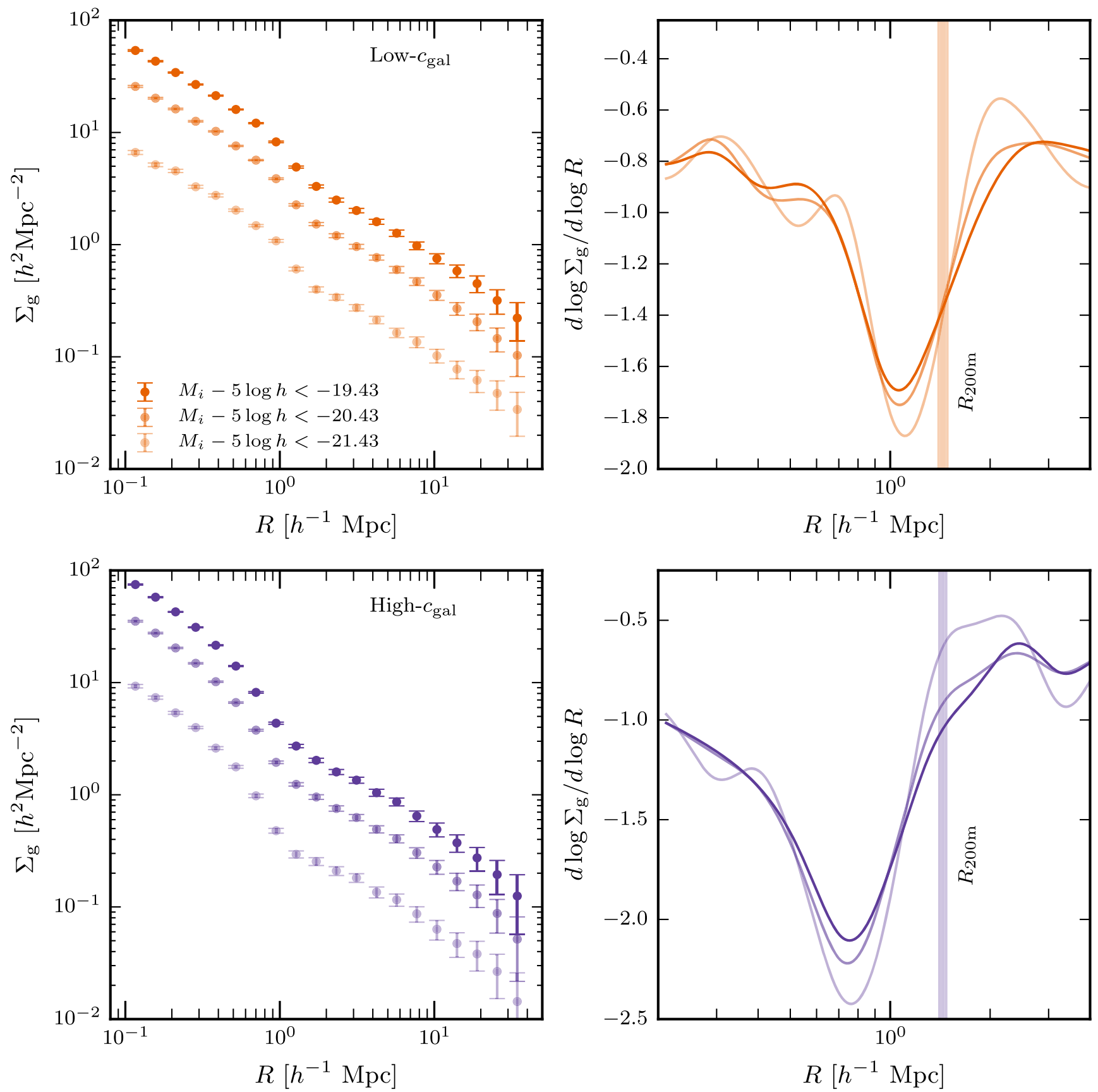

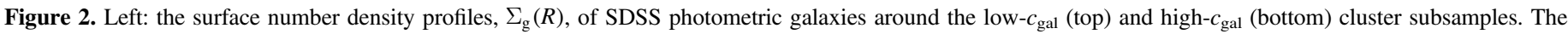

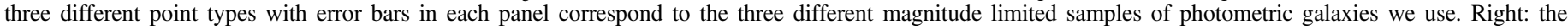

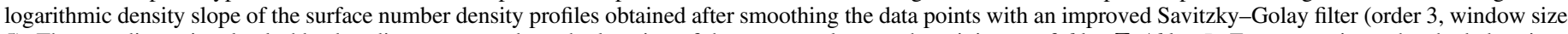

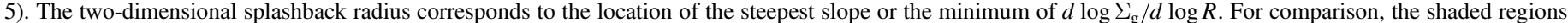

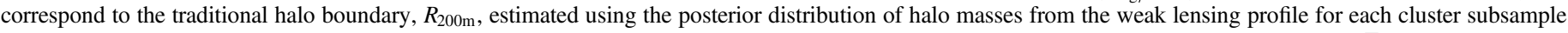

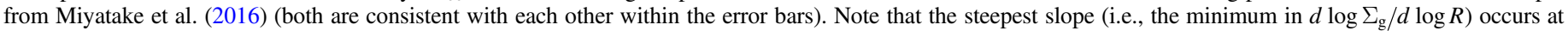
different locations for the two cluster subsamples.

subsamples have different intrinsic clustering biases, as recently reported by Miyatake et al. (2016).

In the left-hand panel of Figure 5, we show the ratio of the number density profiles for our fiducial sample of photometric galaxies around the two subsamples of galaxy clusters on scales of $3-40 h^{-1} \mathrm{Mpc}$. We fit a constant ratio to these measurements accounting for the covariance determined from the jackknife technique. This assumes that the two samples have a similar scale dependence for their bias, and the data support this assumption. The posterior distribution of the constant ratio obtained using this procedure is shown in the right-hand panel of Figure 5. We find a $6.6 \sigma$ deviation of the ratio of the two surface density profiles from unity: $1.48 \pm$ 0.07 . We have thus detected halo assembly bias-the two cluster subsamples have the same halo mass based on weak lensing, but a different large-scale halo bias. For comparison, the difference in the bias ratio that was obtained in Miyatake et al. (2016) using the weak lensing signal was $1.64_{-0.26}^{+0.31}$, and 
Table 1

Posterior Distribution of Parameters from the MCMC Analysis

\begin{tabular}{|c|c|c|c|c|c|c|c|c|c|c|c|c|}
\hline Magnitude & $c_{\mathrm{gal}}$ & $\log \rho_{\mathrm{s}}$ & $\log \alpha$ & $\log r_{\mathrm{s}}$ & $\log \rho_{\mathrm{o}}$ & $s_{\mathrm{e}}$ & $\log r_{\mathrm{t}}$ & $\log \beta$ & $\log \gamma$ & $R_{\mathrm{sp}}^{2 \mathrm{~d}}$ & $R_{\mathrm{sp}}^{3 \mathrm{~d}}$ & $\overline{\chi^{2} / \mathrm{dof}}$ \\
\hline-19.43 & high & $1.10_{-0.77}^{+0.25}$ & $-0.95_{-0.32}^{+0.22}$ & $-0.32_{-0.13}^{+0.40}$ & $0.349_{-0.035}^{+0.031}$ & $1.601_{-0.080}^{+0.076}$ & $-0.082_{-0.040}^{+0.049}$ & $0.762_{-0.095}^{+0.119}$ & $0.66_{-0.12}^{+0.14}$ & $0.778_{-0.014}^{+0.015}$ & $0.971_{-0.021}^{+0.025}$ & $6.0 / 8$ \\
\hline-19.43 & low & $-0.68_{-0.20}^{+0.30}$ & $-1.090_{-0.063}^{+0.088}$ & $0.55_{-0.17}^{+0.11}$ & $0.545_{-0.067}^{+0.055}$ & $1.600_{-0.080}^{+0.060}$ & $0.058_{-0.021}^{+0.023}$ & $1.10_{-0.11}^{+0.12}$ & $0.64_{-0.11}^{+0.13}$ & $1.153_{-0.021}^{+0.029}$ & $1.378_{-0.026}^{+0.026}$ & $13.2 / 8$ \\
\hline-20.43 & high & $0.70_{-0.86}^{+0.31}$ & $-0.97_{-0.35}^{+0.28}$ & $-0.27_{-0.16}^{+0.45}$ & $0.167_{-0.016}^{+0.014}$ & $1.613_{-0.077}^{+0.0074}$ & $-0.098_{-0.038}^{+0.048}$ & $0.82_{-0.11}^{+0.11}$ & $0.69_{-0.12}^{+0.11}$ & $0.756_{-0.012}^{+0.014}$ & $0.938_{-0.026}^{+0.024}$ & $2.9 / 8$ \\
\hline-20.43 & low & $-0.89_{-0.28}^{+0.38}$ & $-1.019_{-0086}^{+0.118}$ & $0.48_{-021}^{+0.15}$ & $0.276_{-0.021}^{+0.018}$ & $1.655_{-0.056}^{+0.052}$ & $0.072_{-0.023}^{+0.026}$ & $1.10_{-0.11}^{+0.12}$ & $0.80_{-0.13}^{+0.14}$ & $1.128_{-0.024}^{+0.029}$ & $1.352_{-0.025}^{+0.026}$ & $12.4 / 8$ \\
\hline-21.43 & high & $0.10_{-0.93}^{+0.39}$ & $-1.00_{-0.35}^{+0.33}$ & $-0.25_{-0.20}^{+0.49}$ & $0.0385_{-0.0048}^{+0.0011}$ & $1.496_{-0.099}^{+0.096}$ & $-0.087_{-0.044}^{+0.051}$ & $0.85_{-0.12}^{+0.14}$ & $0.78_{-0.13}^{+0.13}$ & $0.754_{-0.019}^{+0.024}$ & $0.938_{-0.040}^{+0.036}$ & $10.5 / 8$ \\
\hline-21.43 & low & $-1.31_{-0.37}^{+0.45}$ & $-0.97_{-0.12}^{+0.16}$ & $0.40_{-0.25}^{+0.20}$ & $0.0712_{-0.0066}^{+0.0063}$ & $1.624_{-0.073}^{+0.073}$ & $0.087_{-0.025}^{+0.026}$ & $1.12_{-0.13}^{+0.14}$ & $0.90_{-0.14}^{+0.15}$ & $1.132_{-0.035}^{+0.043}$ & $1.361_{-0.038}^{+0.034}$ & $23.6 / 8$ \\
\hline
\end{tabular}

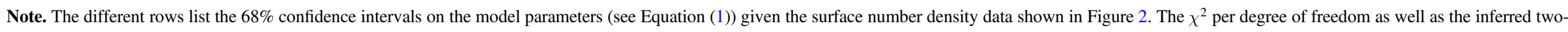
dimensional and three-dimensional splashback radii are also shown in the last three columns. 

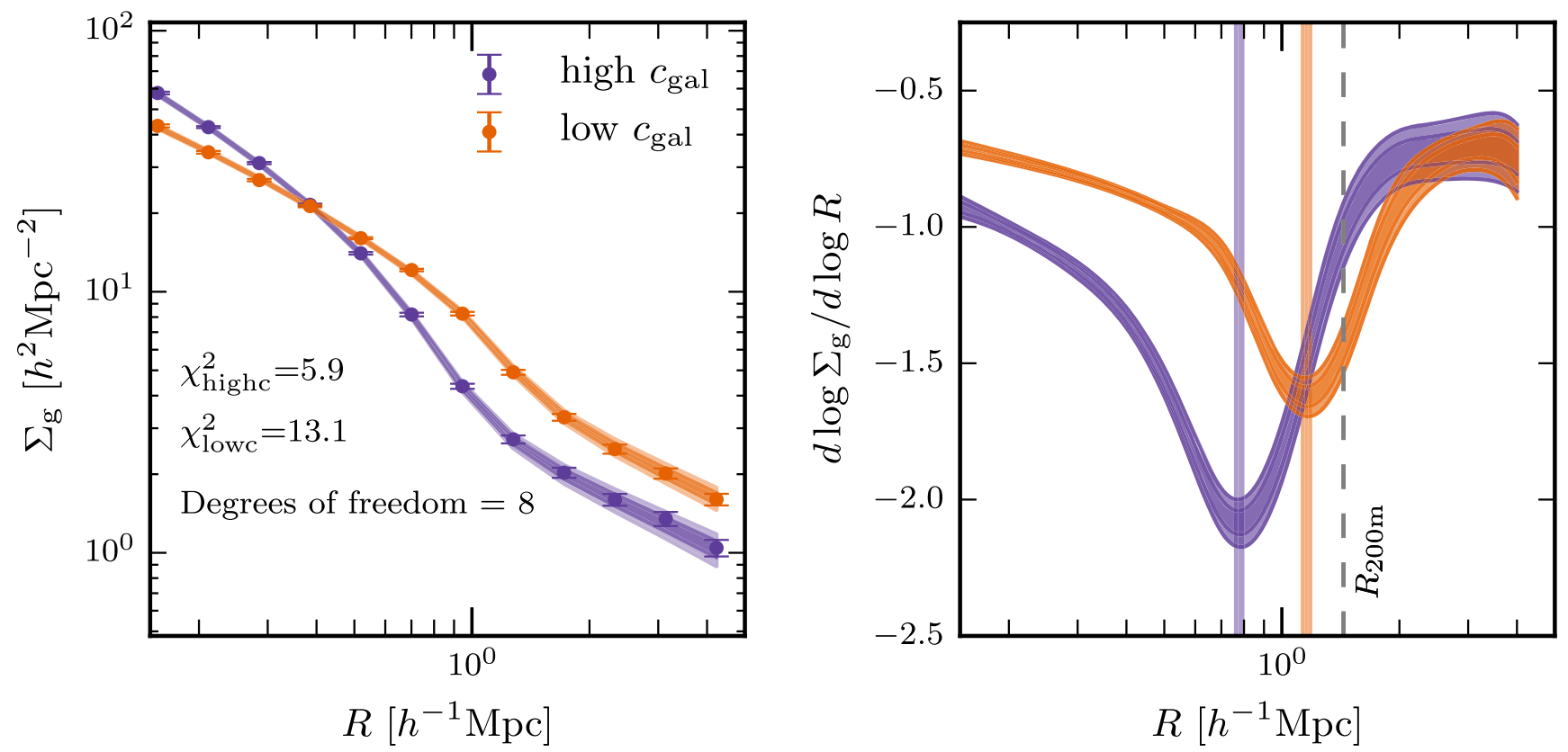

Figure 3. The surface number density profiles, $\Sigma_{\mathrm{g}}(R)$, of our fiducial sample of SDSS photometric galaxies around the two cluster subsamples are shown in the lefthand panel. The shaded regions show the $68 \%$ and $95 \%$ confidence regions of our model fit to the data. The right-hand panel shows the inferred constraints on the logarithmic slope of $\Sigma_{\mathrm{g}}(R)$ for the two subsamples. The splashback radius in two dimensions, $R_{\mathrm{sp}}^{2 \mathrm{~d}}$, corresponds to the location of the steepest slope or the minimum of $d \log \Sigma_{\mathrm{g}} / d \log R$. The $68 \%$ constraints on $R_{\mathrm{sp}}^{2 \mathrm{~d}}$ are marked with vertical shaded regions. These minima occur at significantly different locations for the two cluster subsamples. The traditional halo boundary, $R_{200 \mathrm{~m}}$, is marked by the gray dotted vertical line.

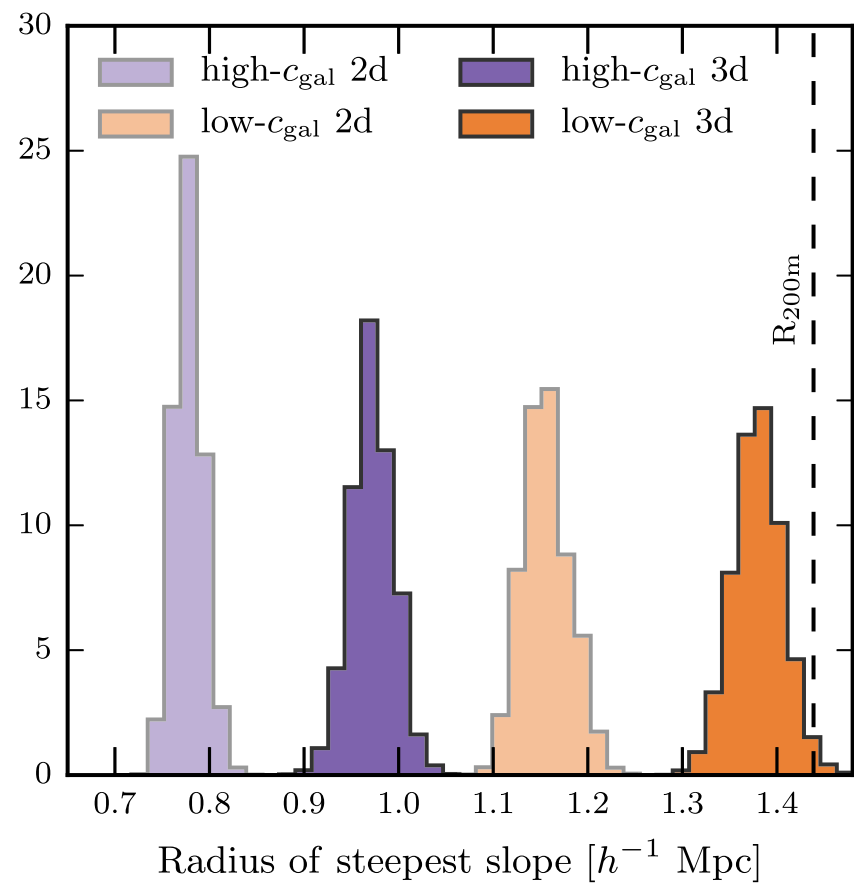

Figure 4. The posterior distributions for the location of the steepest slope of the galaxy density profiles around the high- and low- $c_{\text {gal }}$ cluster subsamples are shown by purple and orange histograms, respectively. The light shaded histograms correspond to the location of the steepest slopes of the surface density profiles (two dimensions), while the dark shaded histograms correspond to the location of steepest slope of the three-dimensional number density profiles inferred by our fits. The locations of the steepest slopes for the two cluster subsamples are significantly different, implying a different mass accretion rate onto these cluster subsamples.

that from the auto-correlation function of galaxy clusters was $1.40 \pm 0.09$. The three different measurements give results which are statistically consistent with each other.

\section{COMPARISONS WITH EXPECTATIONS FROM THE $\Lambda$ CDM MODEL}

Using the projected galaxy number density profiles around two cluster subsamples from the redMaPPer catalog, we have shown that these two subsamples have different profiles, splashback radii, and clustering biases. We now compare these measurements to the predictions of the concordance cosmological $\Lambda$ CDM model.

\subsection{Is the Splashback Radius for the Two Subsamples at the Expected Location?}

Using the weak lensing inferred masses for our cluster subsamples, we can compute the baseline expectation for the location of the splashback radius in the standard cosmological model. The ratio $R_{\mathrm{sp}}^{3 \mathrm{~d}} / R_{200 \mathrm{~m}}$ is expected to depend upon the accretion rate of the halos as well as redshift (Adhikari et al. 2014; Diemer \& Kravtsov 2014; More et al. 2015). To compute the mean accretion rate onto halos, we use halos from MDPL2 at $z=0.248$, closest to the median redshift of our redMaPPer subsamples, and select all halos above a certain halo mass threshold. ${ }^{24}$ We choose the halo mass threshold such that the average halo mass of the sample is consistent with the $M_{200 \mathrm{~m}}$ of the redMaPPer subsamples obtained by Miyatake et al. (2016). As a best-case expectation, we divide the halo sample into two based on the dependence of the halo mass accretion rate on halo mass, $\Gamma\left(M_{200 \mathrm{~m}}\right)=\Delta \log M_{\mathrm{vir}} / \Delta \log a$. The derivative for $\Gamma$ is computed using a finite difference scheme using the virial masses at redshifts 0.248 and 0.748 (Diemer \& Kravtsov 2014; More et al. 2015). ${ }^{25}$

\footnotetext{
$\overline{{ }^{24} \text { Using a cosmological simulation with }} \Omega_{\mathrm{m}}=0.27$, we obtain similar numbers for the expected mass accretion rates.

${ }^{25}$ There is very little difference in the average value of $\Gamma$ if we use a halo mass sample with a threshold on $N_{\text {sat }}$.
} 

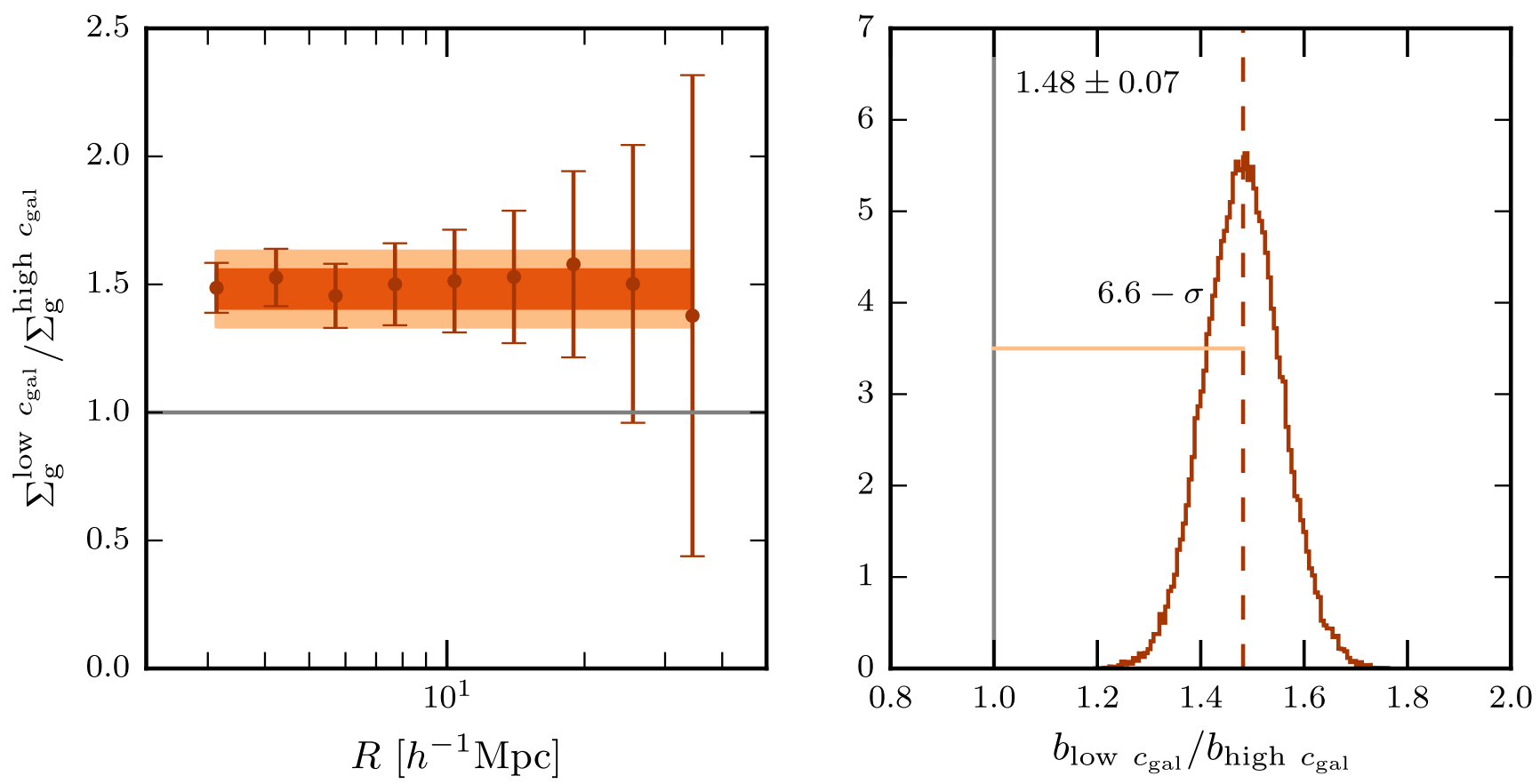

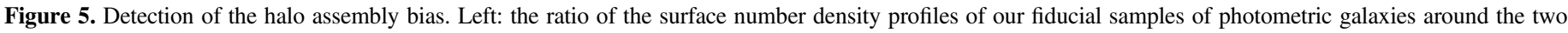

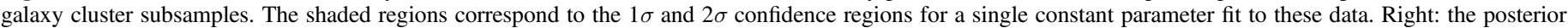

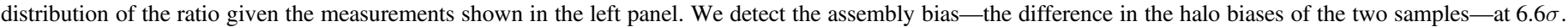
There is a significant covariance in the errors, hence the small point-to-point variation given the errors. The quoted significance accounts for the covariance.

In Figure 6, we compare the location of the splashback radius with respect to $R_{200 \mathrm{~m}}$ observed for our cluster subsamples to the best-case expectations implied by these accretion rates. The gray band corresponds to the fitting function

$$
\frac{R_{\mathrm{sp}}^{3 \mathrm{~d}}}{R_{200 \mathrm{~m}}}=0.58\left[1+0.63 \Omega_{\mathrm{m}}(z)\right]\left(1+1.08 \exp \left[-\frac{\Gamma}{2.26}\right]\right)
$$

with a 5\% uncertainty. This fitting function is a good fit to the splashback radii of dark matter halos in simulations used in More et al. $(2015)^{26}$, but corresponds to the mean profiles instead of the median. The gray star corresponds to the typical expected value of $\Gamma$ for halos in the sample, estimated from the simulations, while the purple and orange stars similarly correspond to the average $\Gamma$ for the best-case simulation subsamples with the fastest and slowest accretion rates (see above). The data seem to prefer a much smaller splashback radius for each of our cluster subsamples $\left(R_{\mathrm{sp}}^{3 \mathrm{~d}} / R_{200 \mathrm{~m}}=0.675_{-0.021}^{+0.024}\right.$ and $0.955 \pm 0.035$ for the highand the low- $c_{\text {gal }}$ subsamples, respectively), even when compared to the splashback radius corresponding to halos with typical $\Gamma$ for our mass scales.

\subsection{Does Dynamical Friction Result in a Smaller Splashback Radius?}

So far in our analysis, we have identified the splashback radius using the galaxy distribution around our cluster subsamples. The splashback radius of galaxies could be different from that of dark matter due to dynamical friction

\footnotetext{
${ }^{26}$ The fitting function was calibrated in the redshift range $[0,4]$.
}

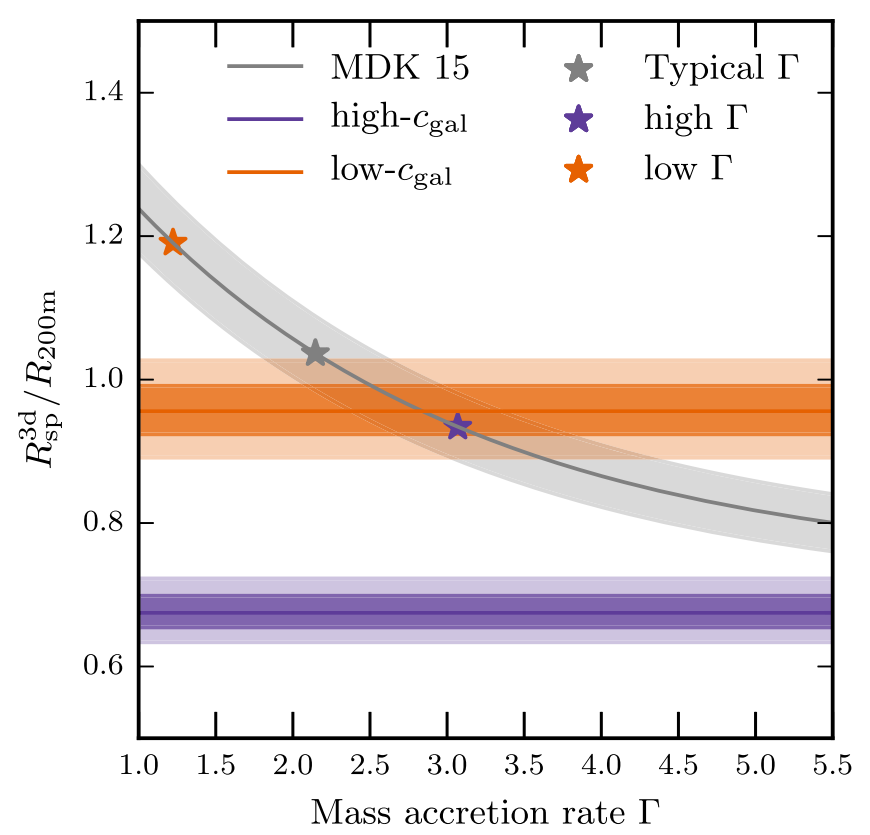

Figure 6. The dependence of $R_{\mathrm{sp}} / R_{200 \mathrm{~m}}$ on the accretion rate $\Gamma$ at $z=0.24$ predicted by $\Lambda \mathrm{CDM}$, shown by a gray line with $5 \%$ uncertainty (More et al. 2015 ). The dark and faint shaded orange (purple) regions display the $68 \%$ and $95 \%$ confidence limits on the splashback radius in three dimensions for our low- (high-) $c_{\text {gal }}$ sample. The gray, orange and purple stars correspond to the typical splashback radii for a typical accretion rate, as well as slow and fast accreting clusters from numerical simulations (see the text for details). The observed values of the splashback radii are significantly smaller than the predicted values from their halo masses, even if we consider halos with typical accretion rates.

acting on the subhalos that host our galaxies, provided these subhalos are sufficiently massive (Adhikari \& Dalal 2016). In what follows, we show that the steepening of the three- 

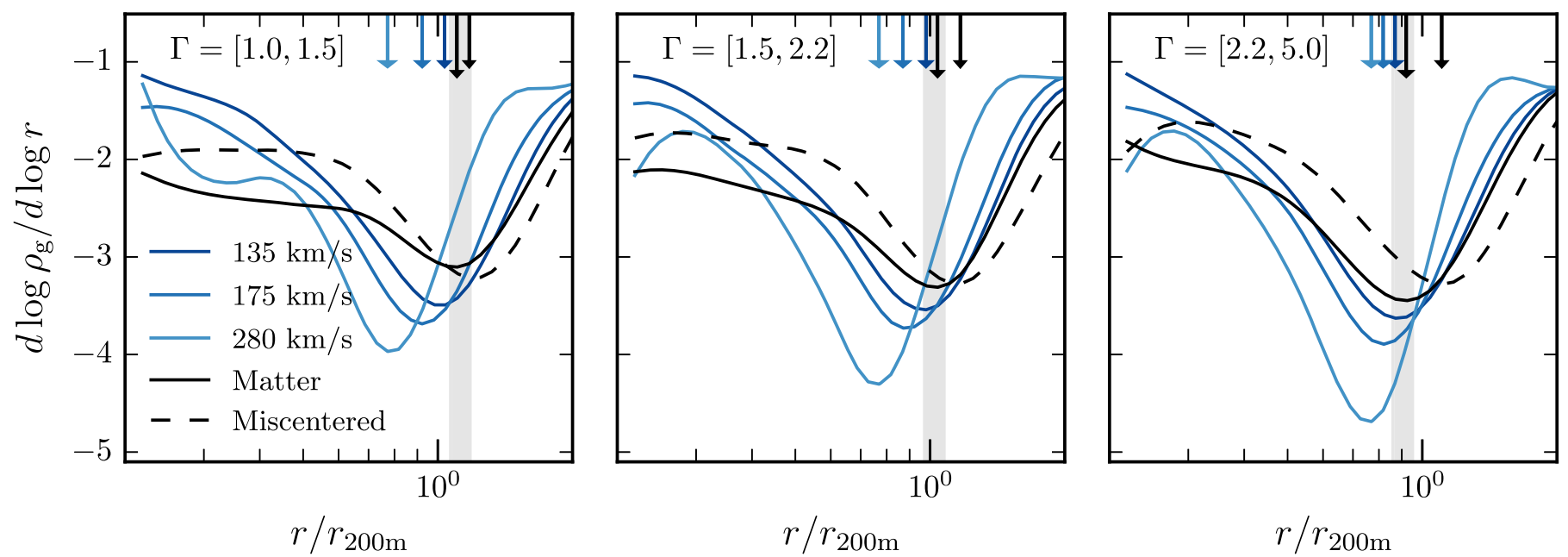

Figure 7. Comparison between the logarithmic slope of the density profile for matter and that of subhalos selected using different $V_{\text {peak }}$ thresholds as indicated in the legend. Different panels correspond to halos with different mass accretion rates, $\Gamma$. The arrows indicate the location of the steepest slope, or the splashback radii for the corresponding population, with the longest arrow used to represent dark matter. The gray vertical bands corresponds to the fitting function for $R_{\mathrm{sp}} / R_{200 \mathrm{~m}}$ similar to the one by More et al. (2015), but for the mean profiles, and includes an uncertainty of $\pm 5 \%$.

dimensional density profiles for both matter and subhalos that host our fiducial photometric sample of galaxies are expected to occur at similar locations.

For this purpose, we again make use of the halo and subhalo catalogs from the MDPL2 simulation. We match the cumulative abundances of dark matter subhalos as a function of $V_{\text {peak }}$ (the maximum circular velocity of a halo throughout its entire history) and that of our photometric galaxies as a function of their magnitude, to obtain an estimate of the $V_{\text {peak }}$ of subhalos hosting our galaxies (see Appendix B). The subhalos that host our fiducial subsample of photometric galaxies approximately correspond to subhalos with $V_{\text {peak }}>135 \mathrm{~km} \mathrm{~s}^{-1}$, while the brighter subsamples correspond to subhalos with $V_{\text {peak }}>175 \mathrm{~km} \mathrm{~s}^{-1}$ and $V_{\text {peak }}>280 \mathrm{~km} \mathrm{~s}^{-1}$, respectively.

For this analysis, we use the $z=0$ particle snapshot of the simulation $^{27}$. We use all halos identified by the six-dimensional phase space halo finder ROCKSTAR (Behroozi et al. 2013) in the $z=0$ snapshot with halo mass, $M_{200 \mathrm{~m}}$, above $8.5 \times 10^{13} h^{-1} M_{\odot}$ as our sample of galaxy clusters. We subdivide these into bins of $\Gamma=\Delta \log M_{\text {vir }} / \Delta \log a$, and compute the three-dimensional density profile of matter around them. The derivative for $\Gamma$ for this particular snapshot was computed to be between $z=0$ and $z=0.5$ (Diemer \& Kravtsov 2014; More et al. 2015). The logarithmic slopes of the matter density profiles around the cluster samples are shown in the different panels of Figure 7 using a black solid line. For reference we also show the expected locations of the splashback radius for each of the subsamples, using Equation (2). The fitting function seems to capture the trend observed for the splashback radius of dark matter as a function of the accretion rate in the simulation reasonably well (within 5\%).

In the same figures we also show the logarithmic slopes of subhalo distributions around galaxy cluster halos for different $V_{\text {peak }}$ thresholds obtained from our simple subhalo abundance matching method. We observe that the locations of the steepest slope for subhalos with the lowest $V_{\text {peak }}$ threshold is similar to that in dark matter within 5\% for all the $\Gamma$ bins shown in the figure. Thus the location of the splashback radius is not

\footnotetext{
${ }^{27}$ Ideally we would have liked to also carry out this exercise near $z=0.24$, but only the $z=0$ particle snapshot was available.
}

expected to be significantly different for subhalos hosting our fiducial sample of photometric galaxies. As we consider $V_{\text {peak }}$ thresholds corresponding to our brighter subsamples, we see the effects of dynamical friction acting on the subhalos (see also Jiang \& van den Bosch 2016). The splashback radius of these larger subhalos systematically shifts to smaller values with increasing $V_{\text {peak }}$ threshold.

We have tried to maximize the effect of dynamical friction in the above exercise by not considering scatter between the luminosity of galaxies and the $V_{\text {peak }}$ of their subhalos while performing abundance matching. We do not see a large shift in the splashback radius of the photometric galaxies around any of our cluster subsamples as a function of their magnitude threshold. However, this does not imply that our data rule out dynamical friction acting on the brighter sample of photometric galaxies. It is quite likely that there is a reasonably large scatter between the magnitude and $V_{\text {peak }}$, which can wash out the dynamical friction effect.

\subsection{Background Subtraction and Mis-centering Uncertainties}

We have used the number density profiles around random points to subtract the uncorrelated galaxies in the background and the foreground of our subsamples. We have also tested how residual uncertainties in background subtraction can affect our results. As an initial test of uncertainties in the background which are constant with radius, we have added in a constant parameter to our model for the projected number density profiles. Even after marginalizing over such a parameter, we obtain values for the splashback radii and its uncertainty which are very consistent in two dimensions, and virtually identical in three dimensions. However, there could be additional background uncertainties which vary with the projected distance.

For example, we expect that the clusters in our subsamples will cause the galaxies in the background to be magnified (see e.g., Umetsu et al. 2011). We explore the changes to the background due to cluster magnification in Appendix D, and find that the splashback radius is not affected even after applying a conservative correction to the background due to the magnification of the clusters. The sky subtraction around bright or highly clustered objects can also potentially affect the 
photometry of galaxies and hence the background objects in clusters (Aihara et al. 2011). This can also partly cancel the magnification effect, as it reduces the number density of background galaxies in clusters.

Mis-centering of central galaxies in redMaPPer clusters could affect the profiles and our estimates of the splashback radius. There are two kinds of mis-centering: first, a galaxy may be mis-classified as central by the cluster finder, and second, the central galaxy may be physically displaced from the potential minimum of the cluster around which all galaxies orbit. To test for the first type of mis-centering, we have restricted our model fits to scales $>400 h^{-1} \mathrm{kpc}$ or to using clusters where the most probable central galaxy has $p_{\text {cen }}>0.9$. These restrictions produce fit parameters consistent with those listed in Table 1, in particular the position of the splashback radius, within the reported uncertainties.

To test for the second kind of mis-centering, we have also considered all halos from the MDLP2 simulation used in the previous section, and displaced $40 \%$ of these halos in their positions with an offset drawn from a multivariate Gaussian distribution with standard deviation equal to $400 h^{-1} \mathrm{kpc}^{28}$ In each panel of Figure 7, we have additionally included a dashed line which shows the slope of the logarithmic density profiles around such a sample of halos. We find that, as expected, in all cases the splashback radius would be overestimated by $\gtrsim 20 \%$, an effect which goes in the opposite direction required to explain a smaller splashback radius. Moreover, the change of slope around the splashback radius is much less pronounced and the overall shape of the profile is significantly modified. This is in contrast to the good agreement we find between the shapes of the predicted and observed slope profiles.

\subsection{Could Averaging Effects Result in a Smaller Splashback Radius?}

We have used the average halo mass of our subsamples as inferred from weak lensing to obtain the average $R_{200 \mathrm{~m}}$ of our cluster halos to compare with the observed values of the splashback radius, $R_{\mathrm{sp}}^{3 \mathrm{~d}}$. Could the difference between the $R_{\mathrm{sp}}^{3 \mathrm{~d}} / R_{200 \mathrm{~m}}$ seen in observations and that predicted from simulations arise due to the finite width of the halo mass distribution? We considered the distribution of halo masses resulting from a threshold sample with the same average halo mass as our cluster subsamples. For such samples, we find that the difference between $\left\langle R_{200 \mathrm{~m}}\right\rangle$ and that inferred from the average halo mass is different by only $\approx 3 \%$, whereas the discrepancy we observe is much larger. ${ }^{29}$

We have also verified that the location of the splashback radius for a threshold mass sample does not result in a smaller inferred splashback radius compared to the expectation based on using the average halo mass, and the average mass accretion rate onto the halo samples. These tests confirm that the smaller value for the splashback radius we observe is not likely to be a result of some averaging effects.

\footnotetext{
${ }^{28}$ This assumes that the redMaPPer centering probabilities are unreliable and the centering algorithm performs as badly as selecting the brightest galaxy in the cluster, which could result in a $40 \%$ mis-centering fraction (Skibba et al. 2011).

${ }^{29}$ Using a halo mass sample with a threshold in $N_{\text {sat }}$, as in our data, also does not affect this conclusion.
}

\subsection{What is the Systematic Error in the Weak Lensing Halo Masses?}

Our conclusion that the observed splashback radius is smaller than the expectation from simulations is based on the comparison with the $R_{200 \mathrm{~m}}$ inferred from the weak gravitational lensing signal by Miyatake et al. (2016). However, Miyatake et al. (2016) assumed a $\delta$-function distribution in halo masses to model the weak lensing measurements for each cluster subsample and infer the average halo mass. Such a simplified fitting to the weak lensing surface mass density profile is expected to underestimate the halo mass by $\sim 10 \%$ compared to the mean halo mass of clusters in the sample (Mandelbaum et al. 2005; Becker \& Kravtsov 2011; Niikura et al. 2015), or $\sim 3 \%$ in radius. The difference between the measured and expected splashback radii is much larger than such a systematic error, and if at all will increase the inconsistency rather than decrease it. The statistical error in the weak lensing masses is less $10 \%$, so even $2 \sigma$ deviations will result in only a $\sim 7 \%$ change in the expectation in the splashback radius. Recently, Simet et al. (2016) also quantified a number of systematic effects related to the weak lensing signal of redMaPPer clusters using the same shape catalog. They also presented toy model estimates for the impact of projection effects on the measured weak lensing halo masses. However all these effects are too small to affect our conclusions. The errors in the weak lensing masses have also been folded in when computing the error on the observed $R_{\mathrm{sp}} / R_{200 \mathrm{~m}}$ shown in Figure 6.

\subsection{Is the Halo Assembly Bias Signal Consistent with Expectations?}

Our observational results indicate that the galaxy cluster subsample with a lower concentration of member galaxies has a larger splashback radius (lower accretion rate $\Gamma$ ), and a larger halo bias. Is the sense and the amplitude of the halo assembly bias signal we see consistent with expectations from cosmological simulations of CDM?

Various proxies such as formation timescales of halos or their dark matter concentrations have been used in the literature to quantify halo assembly bias. The sense of the halo assembly bias effect varies depending upon the assembly proxy used. For example, Gao et al. (2005) find that halo assembly bias is strongest for low mass galaxy scale halos, and that the earliest forming halos cluster more strongly than the average for their halo masses. However, they find that the effect almost disappears on the mass scales we consider in our paper. In contrast, when the concentration of halos is used as a proxy, halo assembly bias manifests itself at both galaxy scales and galaxy cluster scales (Wechsler et al. 2006). At galaxy scales (masses below $10^{12} h^{-1} M_{\odot}$ ) high concentration halos (which form earlier) have a larger halo bias, but the trend reverses on galaxy cluster scales, as expected from the relation between mass accretion history and the curvature of initial density peaks (Dalal et al. 2008).

Li et al. (2008) explored eight different definitions of halo assembly and found that the connection between formation time and assembly bias of halos can be totally washed out or even reversed depending upon the proxy used. Member galaxy concentration has never been explored previously in the literature as a proxy for assembly history. Therefore, we use the MDPL2 simulation to explore the extent of the assembly bias expected when using it as a proxy. We ignore all 
observational complications, and investigate how the clustering of halos at fixed halo mass varies as a function of the concentration of the subhalo distribution belonging to the halos.

We use all isolated halos with $M_{200 \mathrm{~m}}>10^{14} h^{-1} M_{\odot}$ at $z=0.248$ from MDPL2. This threshold in halo mass at $z=0.248$ allows us to match the average weak lensing mass of our cluster subsamples. ${ }^{30}$ To compute the average projected separation between cluster members and halo centers, we use all subhalos with $V_{\text {peak }}>135 \mathrm{~km} \mathrm{~s}^{-1}$, similar to the threshold used for the faintest of our photometric galaxies. We divide the sample into halves based on the three-dimensional clustercentric distance of the subhalos of each cluster as shown in the top left panel of Figure 8. The top right panel of the figure shows the scatter plot of dark matter concentrations of our halos given their halo masses, and implies that the concentration of dark matter and the concentration of subhalos are not well correlated.

The ratio of the halo bias obtained from the auto-correlation function of cluster halos in the two subsamples is shown in the bottom left panel of Figure 8 with the orange solid line, while that obtained from the cross-correlation with halos with $V_{\text {peak }}>135 \mathrm{~km} \mathrm{~s}^{-1}$ is shown with a dashed line. For comparison, the observed ratio between the biases of our cluster subsamples is shown with the gray shaded region. Subsample divisions based on $\left\langle R_{\text {mem }}^{3 \mathrm{~d}}\right\rangle\left(N_{\text {sat }}^{3 \mathrm{~d}}\right)$ give a very similar amplitude for this ratio at a larger radius when using auto-correlations, but with weaker scale dependence. Finally, the bottom right panel shows the distributions of the mass accretion rates onto these clusters.

The halo assembly bias signal we observe in the data is similar, albeit stronger, than that seen in simulations. ${ }^{31}$ The division in the mass accretion rates is also as required to explain the splashback radius measurements seen in the data. These results from simulations show that it is possible to obtain samples of halos with a lower average accretion rate that have a larger clustering signal, as seen in our observations.

Once projection effects are accounted for (as in Section 4.7), and the sample is divided based upon $\left\langle R_{\mathrm{mem}}^{2 \mathrm{~d}}\right\rangle\left(N_{\mathrm{sat}}^{2 \mathrm{~d}}\right)$, an even smaller value for the halo assembly bias signal is obtained in simulations. Given that the strength of the halo assembly bias signal increases with halo mass, one could potentially reproduce the result by using larger mass scales in simulations. However this will further worsen the problem of the smaller splashback radius. Characterization of the halo assembly bias signal with different richness threshold samples and theoretical investigations using larger simulations are currently ongoing and will be reported in a future publication.

\subsection{Projection Effects}

One possible way to explain the different splashback radii and halo biases for our two cluster subsamples could be that the two SDSS cluster subsamples in reality have different masses. The weak lensing mass estimates, taken at face value, restrict

\footnotetext{
${ }^{30}$ Note that this threshold is slightly larger than the threshold used used in Section 4.2 at $z=0.0$. Both thresholds ensure that we match the average weak lensing mass from observations.

31 The preliminary investigations mentioned in Miyatake et al. (2016), which seemed to suggest larger assembly bias signal, were mistakenly performed using a larger mass threshold. The results presented in this paper override those preliminary investigations.
}

this possibility. However, the weak lensing signal is only sensitive to the projected mass distribution. The cluster subsample identification also has a very poor resolution in the line of sight direction. Therefore, we explore the possibility that our subsamples indeed have different three-dimensional masses regardless of the similarity of the weak lensing signal and the richness of the clusters.

To address the magnitude of projection effects, we again resort to the MDPL2 simulation. We consider all halos with $M_{200 \mathrm{~m}}>10^{13} h^{-1} M_{\odot}$ as potential clusters identified from the photometric data. ${ }^{32} \mathrm{We}$ associate all halos/subhalos with $V_{\text {peak }}>135 \mathrm{~km} \mathrm{~s}^{-1}$ to be satellite galaxies of these potential galaxy clusters if they lie within a projected radius of $R_{200 \mathrm{~m}}$ from their centers, and have a line of sight separation less than $50 h^{-1} \mathrm{Mpc}$. The projected separation lengths of $\pm 50 h^{-1} \mathrm{Mpc}$ were obtained by considering the scatter in the colors of redsequence galaxies used to identify redMaPPer galaxy clusters, and the amount of variation of these colors as a function of redshift (see Figures 1 and 7 in Rykoff et al. 2014). If subhalos can belong to two clusters after the projection, we assign them to the most massive halo. We do not redefine or recompute the cluster centers after these projections as we expect that the redMaPPer centering algorithm is less likely to identify the galaxies in projection as centrals for the redMaPPer clusters.

In the left-hand panel of Figure 9, we show the scatter plot of $M_{200 \mathrm{~m}}^{3 \mathrm{~d}}$ as a function of $N_{\text {sat }}^{2 \mathrm{~d}}$, i.e., the number of satellites associated with these cluster-sized halos after the reassignment described above. To maximize the strength of the projection effect, we make the extreme assumption that our two cluster subsamples select the upper and the lower half of $M_{200 \mathrm{~m}}^{3 \mathrm{~d}}$ at fixed $N_{\mathrm{sat}}^{2 \mathrm{~d}}$. The distributions of the $M_{200 \mathrm{~m}}^{3 \mathrm{~d}}$ selected in this manner are displayed in the right-hand panel of Figure 9. The average halo masses corresponding to the two distributions are different by $\sim 62 \%$, which implies a difference of at most $\sim$ $20 \%$ in the radii. In contrast, the observed difference in the radii is twice as large, $\sim 40 \%$. We expect the extent of projection effects to be lower, given that the average cluster-centric distances for the satellites are not expected to be perfectly correlated with $M_{200 \mathrm{~m}}^{3 \mathrm{~d}}$ at fixed $N_{\text {sat }}^{2 \mathrm{~d}}$. Furthermore, due to halo assembly bias effects in simulations, the sample with larger $M_{200 \mathrm{~m}}^{3 \mathrm{~d}}$ at fixed $N_{\text {sat }}^{2 \mathrm{~d}}$ turns out to have a smaller clustering signal, opposite to what is required to simultaneously explain both the different splashback radius and the sense of the assembly bias signal.

Thus, we have established that the two subsamples have splashback radii and halo biases that differ from one another by an amount that cannot be explained with the help of projection effects alone. In Appendix C, we show that both of our cluster subsamples have very similar distributions of the axis ratios of the light profiles for the most probable central galaxies, similar ellipticity distributions for satellite galaxies, and similar line of sight velocity dispersions. The similarity of these distributions is inconsistent with the trends expected, if the projection effects were behind the large magnitude of assembly bias signal or the difference in the splashback radii between the two subsamples divided by $\left\langle R_{\mathrm{mem}}\right\rangle$.

\footnotetext{
32 We have chosen a slightly lower halo mass threshold for this test since projection effects can upscatter smaller halos to have a larger richness than expected for their halo masses.
} 

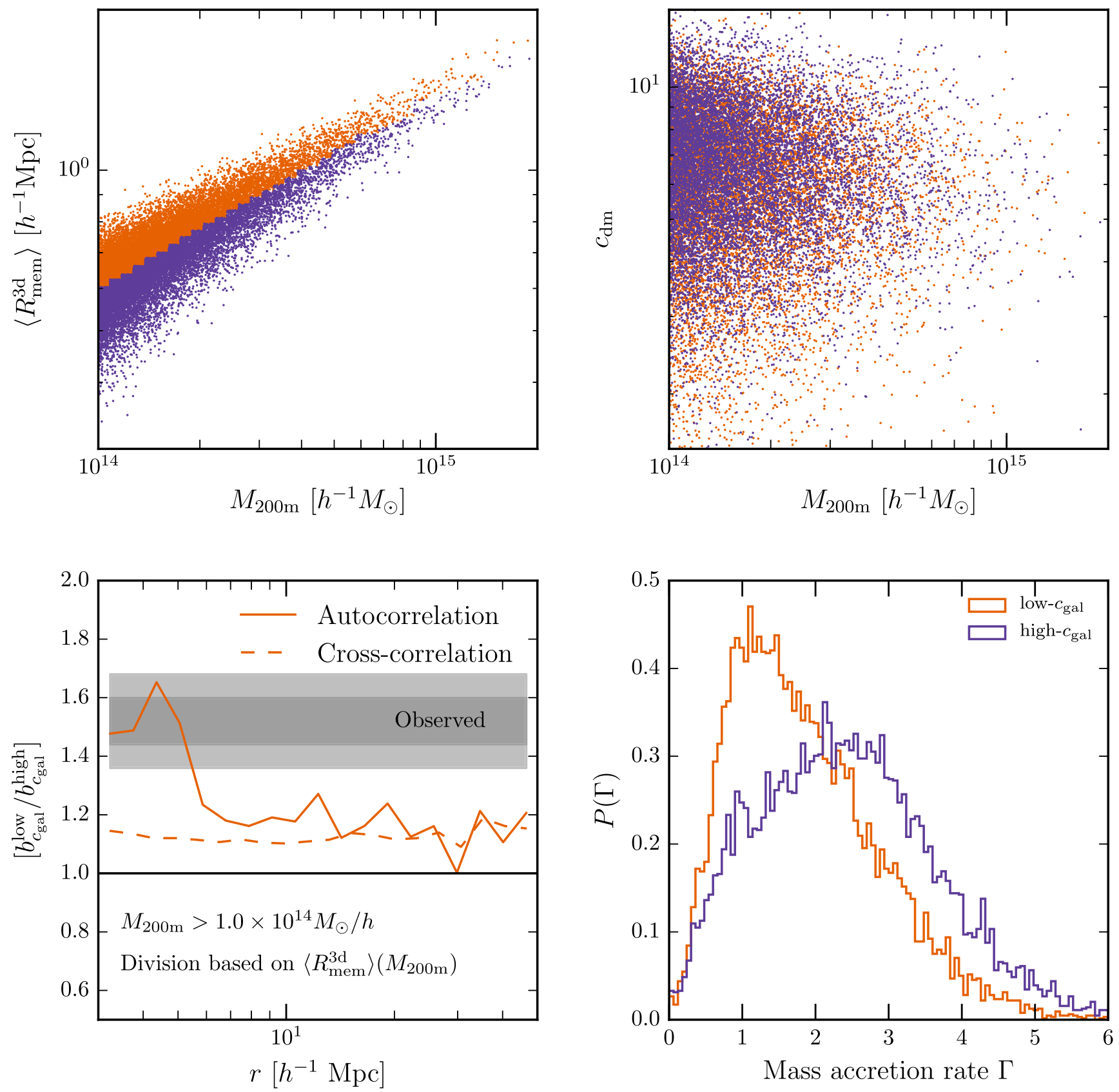

Figure 8. Expectation for halo assembly bias from simulations. Top left: the average cluster-centric distance of member subhalos in cluster-sized halos in the MDPL2 simulation as a function of halo mass. We use the radius to divide the cluster sample into two at a fixed mass. The two subsamples are shown in orange and purple. Top right: the same subsamples as in the top left panel but in the dark matter concentration-halo mass plane. The subhalo distribution seems to have very little correlation with the dark matter distribution. Bottom left: the ratio of the halo biases of subsamples of halos with masses $10^{14} h^{-1} M_{\odot}$ split using the average distance of their subhalos from their centers, $\left\langle R_{\text {mem }}^{3 \mathrm{~d}}\right\rangle\left(M_{200 \mathrm{~m}}\right)$. The bias ratio obtained from cluster--cluster auto-correlations is shown with a solid line, while the dashed line corresponds to the ratio obtained from the cross-correlations of cluster-scale halos with all subhalos with $V_{\text {peak }}>135 \mathrm{~km} \mathrm{~s}^{-1}$, which are selected to mimic the fiducial photometric galaxies used in our analysis as in Figure 1. Bottom right: the distribution of the mass accretion rates $\Gamma$ for the two subsamples.

\section{DISCUSSION}

In Section 3, we showed that the surface number density profiles of galaxies around redMaPPer clusters exhibit steepening characteristic of the splashback radius expected to arise in dark matter halos due to the caustic formed by matter at the first apocenter after accretion. Moreover, we showed that subsamples of the clusters of the same mass split on concentration of the galaxy distribution exhibit different splashback radii and different spatial biases.
Although comparisons with the theoretical expectations presented in Section 4 show that the observed profiles and trends agree with the predictions qualitatively, there are significant quantitative discrepancies. First, the observed splashback radii are significantly smaller than expected for halos corresponding to the cluster masses of our sample, and second, the difference in the biases of cluster subsamples is also larger than expected. Both discrepancies are very interesting because the location of the splashback radius is a result of simple gravitational dynamics and can be both 

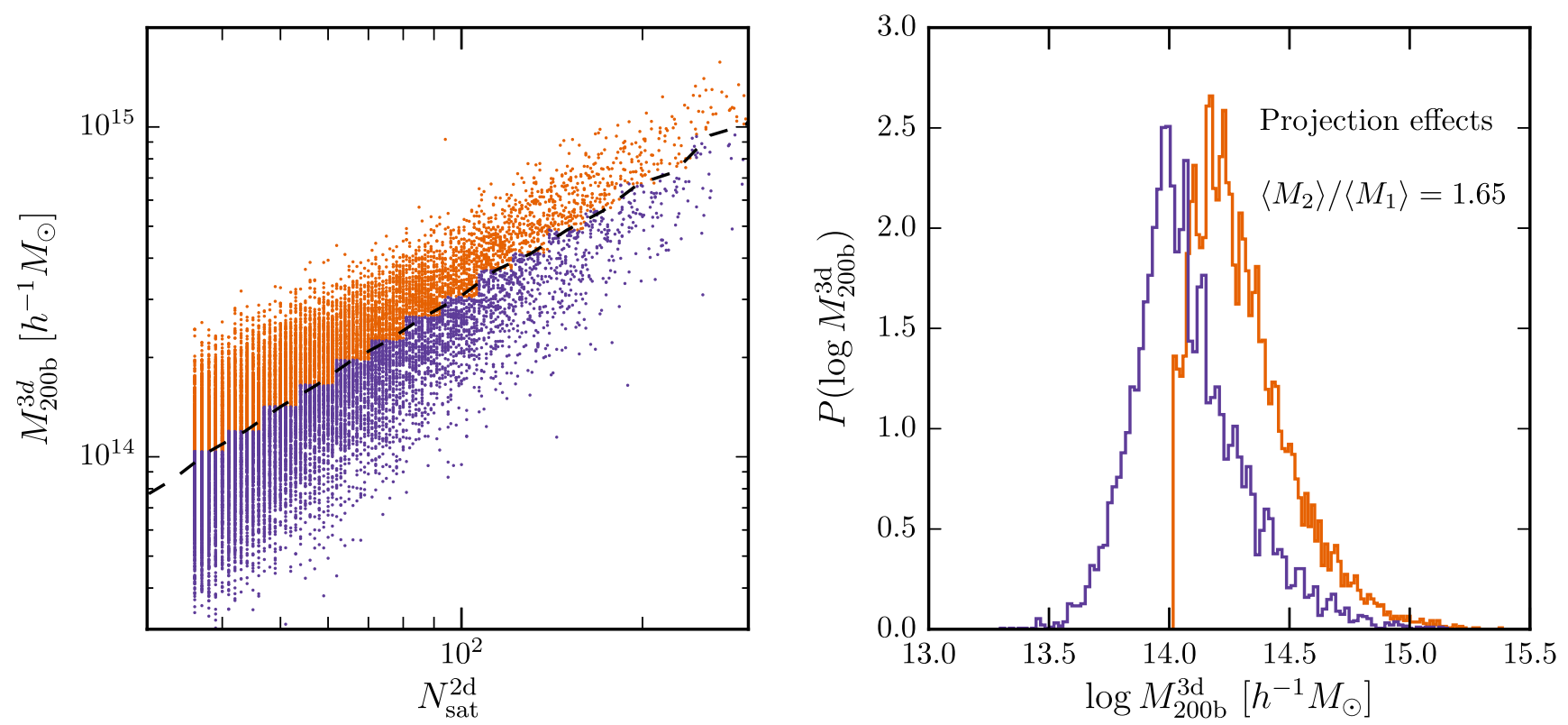

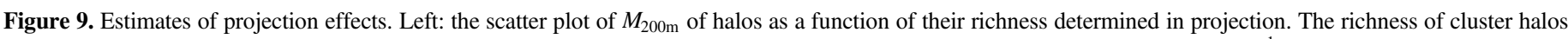

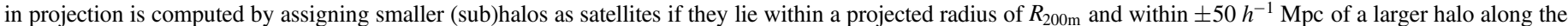

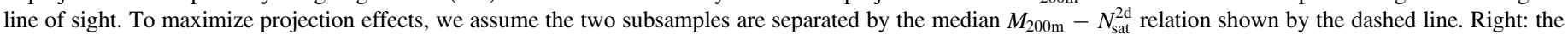

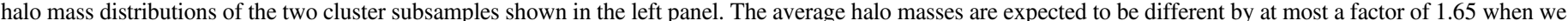
maximize the projection effects.

robustly predicted from simulations (Diemer \& Kravtsov 2014; More et al. 2015) and reproduced even in simple spherical collapse models (Adhikari et al. 2014). Likewise, halo assembly bias is expected to reflect the statistics of the peaks in the initial density field from which massive cluster-sized halos form (Dalal et al. 2008). We will postpone the discussion of the larger magnitude of halo assembly bias to a future paper where we will quantify with larger simulations the extent of this discrepancy. We focus on the splashback radius discrepancy below.

We consider four possible explanations for the discrepancies in the splashback radius: (a) the halo mass $M_{200 \mathrm{~m}}$, and hence the radius $R_{200 \mathrm{~m}}$ inferred from the data, is larger than it really is, and this causes our measurements of $R_{\mathrm{sp}} / R_{200 \mathrm{~m}}$ to be biased low; (b) the splashback radius of the clusters for a given accretion rate measured using galaxies is smaller than that expected from dark matter simulations; (c) the mass accretion rate onto clusters is much higher than expected for clusters of this mass; and, finally, (d) that new physics in the form of selfinteractions of dark matter particles results in a decrease of the splashback radius. We discuss each of these possibilities in turn.

The ratio between the splashback radii of the two cluster subsamples is roughly consistent with the best-case expectations from the $\Lambda \mathrm{CDM}$ simulation of the Planck cosmology (the ratio between the splashback radii corresponding to the orange and purple stars in Figure 6). Let us assume that our observational proxy results in the best-case split, a very optimistic assumption, and that the weak lensing masses are overestimated. In that case, the discrepancy seen in the splashback radii for the two cluster subsamples is $\sim 1.4$ in radii, thus a factor of $\sim 2.75$ in halo masses, strongly disfavored by the small errors on the masses from weak lensing. Under this scenario, we would have uncovered a critical problem for weak lensing calibration of observable halo mass relations which are important for precision cosmological measurements using galaxy clusters. Moreover, even if we assume that the masses are overestimated for both samples, this would mean that the predicted assembly bias strength would be even lower. Such arguments thus run in the opposite sense of what is required to explain the large assembly bias signal.

If weak lensing systematics can be ruled out, one could try to explain the discrepancy if the mass accretion rate onto the clusters is normal, but the splashback radius is indeed smaller than expected. Dynamical friction on subhalos is an obvious physical effect which could cause this. However, in Section 4.2, we have shown that the subhalos corresponding to the faintest magnitude bin we consider are not expected to experience a large amount of dynamical friction. Also, puzzlingly, in the data we do not detect a significant difference in the splashback radius with the brightness of the photometric sample used. Furthermore, future deeper surveys should be able to rule out this possibility by using even fainter galaxies. Neutrinos with masses or warm dark matter scenarios consistent with the current constraints also do not result in a large change in the splashback radius (Villaescusa-Navarro \& Dalal 2011; Villaescusa-Navarro et al. 2013). Other astrophysical possibilities include subtle biases in the photometric galaxy samples, which lead to a preferential selection of slow moving galaxies in our sample. For example, ram pressure stripping is expected to be more effective in stripping out gas and shutting down the star formation in fast moving galaxies, thus removing them preferentially from our photometric sample. Detection of the splashback radius of matter in clusters using the weak lensing signal would be able to test this possibility.

Another possibility could be that the cosmological parameters of the universe are different from the fiducial ones we have assumed for the data analysis in this paper. Let us assume, for example, that the universe has $\Omega_{\mathrm{m}}=0.33$, a $\sim 20 \%$ larger value compared to $\Omega_{\mathrm{m}}=0.27$ assumed in this paper. In such a case, the weak lensing signal is affected by two competing factors: the change in the critical density of the universe, used 
to infer $\Delta \Sigma(R)$ from the observable weak lensing distortion, and the change in the conversion from angular coordinates to comoving coordinates (More 2013). These two factors roughly cancel each other, leaving the inferred comoving density profile around the clusters approximately unchanged (More 2013). Nevertheless, the $R_{200 \mathrm{~m}}$ inferred from the density profiles would be smaller by about $7 \%$ since the definition of $R_{200 \mathrm{~m}}$ is tied to the cosmological mean matter density, such that $R_{200 \mathrm{~m}} \propto \Omega_{\mathrm{m}}^{-1 / 3}$, approximately. The change in the conversion of angles to comoving distances will reduce the location of the observed splashback radius by about $1 \%$. Overall this will result in an increase of the observed $R_{\mathrm{sp}} / R_{200 \mathrm{~m}}$ ratio by about $5 \%-6 \%$. However, because the expected value of $R_{\mathrm{sp}} / R_{200 \mathrm{~m}}$ also depends on $\Omega_{\mathrm{m}}$ (Adhikari et al. 2014; Diemer \& Kravtsov 2014; More et al. 2015), this larger observed value will have to be compared to a $4 \%$ larger theoretical expectation. Thus a change to the cosmological parameters in the context of a flat $\Lambda \mathrm{CDM}$ model is not expected to significantly reduce the extent of the observed discrepancy.

Dark matter self-interactions have long been proposed to alleviate problems on small scales in the standard cosmological model (see e.g., Spergel \& Steinhardt 2000). Under certain conditions, discussed below, the drag force due to interactions between dark matter particles of subhalos and cluster halos could lead to loss of orbital energy by subhalos even on the first crossing, thereby reducing the splashback radius.

For isotropic elastic scattering, we do not expect dark matter self-interactions to significantly affect the splashback feature, because the upper limits on such an interaction cross-section are sufficiently stringent to ensure that most dark matter particles do not experience any scattering events during a single orbit (Gnedin \& Ostriker 2001; Randall et al. 2008). Of the few subhalo particles that do scatter, most are ejected from their subhalos, since the orbital velocities of subhalos within massive hosts are typically larger than the internal escape velocities of those subhalos. Therefore we would expect evaporation of subhalo masses, without a significant drag for isotropic scattering.

On the other hand, if dark matter self-interactions are anisotropic, with large cross-sections for small angle scattering and low cross-sections otherwise, then the momentum transfer during dark matter interactions may not necessarily be large enough to ensure ejection. The small angle scattering crosssections could then be large enough for dark matter particles to experience frequent interactions and yet obey the bounds on subhalo evaporation. The subhalos would experience a net deceleration given by

$$
d=\frac{\rho(r, t) v(t)^{2} \sigma_{\mathrm{tr}}}{2 m_{\mathrm{dm}}}
$$

where $v(t)$ is the relative velocity of the subhalo, $\rho(r, t)$ is the time-dependent density profile of the cluster halo, $m_{\mathrm{dm}}$ is the mass of the dark matter particle, and $\sigma_{\text {tr }}$ is the momentum transfer cross-section (Kahlhoefer et al. 2014).

We have carried out simple analytical calculations based on a spherical collapse model similar to Adhikari et al. (2014; see also Adhikari \& Dalal 2016), but including a velocitydependent drag term of the above form. We find that the momentum transfer cross-section required to reduce the splashback radius by $\approx 20 \%$ can range from 1 to $10 \mathrm{~cm}^{2} \mathrm{~g}^{-1}$ depending upon the pericenter of accreting halos on their first passage through the halo (S. More 2016, in preparation). The ambient dark matter density, and the relative velocity, hence the resultant drag, reach a maximum at the pericenter. Therefore, a proper treatment of the orbital parameters of subhalos expected in the standard structure formation model is required to determine the effects of dark matter self-interactions on the splashback radius (Jiang et al. 2014). We defer such investigations to a future paper.

Although the existing constraints on such scenarios are pretty weak, the recent discovery of galaxy displacement with respect to its subhalo in one of the clusters (Harvey et al. 2015) could be a signature of self-interaction (with a cross-section consistent with that required to explain $R_{\text {sp }}$ discrepancy, see Kahlhoefer et al. 2015). Numerical simulations of this type of dark matter self-interaction, similar to the simulations performed for hard-sphere interactions (Elbert et al. 2015), would be required to refine the estimate of the cross-sections stated above further.

Note that even if the self-interactions will ultimately not turn out to be the explanation for the splashback radius discrepancy, our analysis shows that precise measurements of galaxy distributions in clusters could provide valuable and competitive constraints on the cross-section of dark matter self-interaction.

If we assume that the differences in the splashback radius we find are not due to the above possibilities and we trust the simple dynamics within the gravitational potential of halos, then our measurements of the smaller splashback radius would either require a different phase space structure in the outskirts of cluster halos or extreme mass accretion rates onto our cluster subsamples. The former possibility requires the velocities of material in the infall streams to be about $25 \%-30 \%$ smaller. For the latter possibility, our parent sample of clusters requires values of $\Gamma \sim 4$, while the high- $c_{\text {gal }}$ sample prefers values of $\Gamma$ $\sim 4$. This would represent a serious challenge to the standard cosmological model. Whether modifications to gravity could achieve such values, while still obeying the bounds from galaxy cluster abundances, needs further careful evaluation (Adhikari et al. 2016).

\section{SUMMARY}

We have used SDSS redMaPPer galaxy clusters and photometric galaxies around them to observationally investigate the boundaries of galaxy clusters, and their relation to assembly history and to halo assembly bias on galaxy cluster scales. For this purpose, we have considered two cluster subsamples defined in Miyatake et al. (2016) which share identical richness and redshift distributions, but have different internal distributions of cluster members. These subsamples were shown to have similar halo masses as inferred from weak gravitational lensing, but have different large-scale biases as measured from their large-scale weak lensing and autocorrelation signals (Miyatake et al. 2016). Our results can be summarized as follows:

1. We detect the surface number density profiles of SDSS photometric galaxies with ${ }^{0.1} M_{i}-5 \log h<-19.43$ around both our cluster subsamples. The surface densities show a sharp steepening around scales of $1 h^{-1} \mathrm{Mpc}$.

2. We modeled the two surface density profiles using the profile advocated by Diemer \& Kravtsov (2014), to infer the location of the steepening in projected and real space for both cluster subsamples. The steepening of the surface density profiles occurs at significantly different locations 
for the two cluster subsamples. We interpret the steepening as the location of the splashback radius for these galaxy clusters. We attribute the difference in the splashback radii for the cluster subsamples to being the result of different accretion rates onto the cluster subsamples. This implies a different assembly history for the two cluster subsamples.

3. Using simple subhalo abundance matching, we investigated whether dynamical friction affects the location of the splashback radius of the subhalos expected to host our galaxies compared to that of dark matter. For the fiducial subsample, we found that the two radii should coincide within 5\%. Observationally, the location of the steepening of the galaxy density profiles for the two cluster subsamples does not change significantly, even for photometric galaxies one or two magnitudes brighter than our fiducial sample.

4. We showed that the amplitudes of clustering of photometric galaxies around our two subsamples of galaxy clusters are different by $6.6 \sigma$. We have thus detected halo assembly bias, a difference in the clustering amplitude of cluster-scale halos with the same mass and different assembly histories.

5. We showed that projection effects could at most cause the two subsamples to have masses different by a factor of 1.6, which is smaller than the difference required to explain the difference in the splashback radii of the two subsamples, or the different halo biases.

6. Using a large cosmological $\Lambda \mathrm{CDM}$ simulation, we have shown qualitative agreement between the trends in the splashback radius and halo assembly bias, as seen in observations. However, the splashback radii of the two cluster subsamples seem to be smaller, while the assembly bias effect larger, than naive expectations. The tests presented in the paper show that none of the systematics are large enough alone to resolve the discrepancies. If astrophysical systematics related to weak lensing, optical cluster finding, and projection effects can be conclusively ruled out, it will imply either a discrepancy between the observed accretion rates onto clusters from the expected ones, or may hint at possible interactions in the dark matter sector, both remarkably interesting possibilities.

Funding for the SDSS and SDSS-II has been provided by the Alfred P. Sloan Foundation, the Participating Institutions, the National Science Foundation, the U.S. Department of Energy, the National Aeronautics and Space Administration, the Japanese Monbukagakusho, the Max Planck Society, and the Higher Education Funding Council for England. The SDSS website is www.sdss.org/.

The SDSS is managed by the Astrophysical Research Consortium for the Participating Institutions. The Participating Institutions are the American Museum of Natural History, Astrophysical Institute Potsdam, the University of Basel, the University of Cambridge, Case Western Reserve University, the University of Chicago, Drexel University, Fermilab, the Institute for Advanced Study, the Japan Participation Group, Johns Hopkins University, the Joint Institute for Nuclear Astrophysics, the Kavli Institute for Particle Astrophysics and Cosmology, the Korean Scientist Group, the Chinese Academy of Sciences (LAMOST), Los Alamos National Laboratory, the
Max-Planck-Institute for Astronomy (MPIA), the Max-PlanckInstitute for Astrophysics (MPA), New Mexico State University, Ohio State University, the University of Pittsburgh, the University of Portsmouth, Princeton University, the United States Naval Observatory, and the University of Washington.

The CosmoSim database used in this paper is a service by the Leibniz-Institute for Astrophysics Potsdam (AIP). The MultiDark database was developed in cooperation with the Spanish MultiDark Consolider Project CSD2009-00064. The MultiDark-Planck II (MDPL2) simulation has been performed in the Supermuc supercomputer at LRZ using time granted by PRACE.

We thank the referee for the positive report and recommendations of additional discussion items. We acknowledge useful discussions with Simon White, Frank van den Bosch, Andrew Hearin, Andrew Zentner, Erik Tollerud, Shigeki Matsumoto, Hitoshi Murayama, Justin Khoury, Mark Trodden, Bhuvnesh Jain, Daisuke Nagai, Uros Seljak, Susmita Adhikari, Xun Shi, and Arka Banerjee. M.T. and S.M. are supported by the World Premier International Research Center Initiative (WPI Initiative), MEXT, Japan, and by the FIRST program "Subaru Measurements of Images and Redshifts (SuMIRe)", CSTP, Japan. S.M., M.T., and M.O. are also supported by Grant-inAid for Scientific Research from the JSPS Promotion of Science (No. 15K17600, 16H01089, 23340061, 26610058 and 26800093), MEXT Grant-in-Aid for Scientific Research on Innovative Areas (No. 15H05893, 15K21733, 15H05892), and by JSPS Program for Advancing Strategic International Networks to Accelerate the Circulation of Talented Researchers. H.M. is supported in part by the Japan Society for the Promotion of Science (JSPS) Research Fellowships for Young Scientists and by the Jet Propulsion Laboratory, California Institute of Technology, under a contract with the National Aeronautics and Space Administration. A.K. was supported by by the Kavli Institute for Cosmological Physics at the University of Chicago through grant PHY-1125897 and an endowment from the Kavli Foundation and its founder Fred Kavli. Ry.M. acknowledges financial support from the University of Tokyo-Princeton strategic partnership grant. R. M. acknowledges support from the Department of Energy Early Career Award program.

\section{APPENDIX A}

\section{TESTING METHODS USING SIMULATIONS}

In this appendix, we use the MDPL2 simulation to test our analysis methods. In particular, we show that we can recover the splashback radius for subhalos in three dimensions using the surface density profiles of subhalos around cluster-scale halos.

We use all halos or subhalos in the halo catalogs with $V_{\text {peak }}>135 \mathrm{~km} \mathrm{~s}^{-1}$ at $z=0 .^{33} \mathrm{We}$ compute the projected surface density of these subhalos around halos with masses $M>8.5 \times 10^{13} h^{-1} M_{\odot}$, where the threshold was chosen to obtain a sample of halos which share the average weak lensing mass of the redMaPPer clusters we use in this paper. ${ }^{34} \mathrm{We}$ subdivide the sample into two subsamples with different mass accretion rates, $\Gamma$, using the median mass accretion rate-halo mass relation $\Gamma(M)$.

\footnotetext{
33 See Appendix B for our justification to choose a threshold of $135 \mathrm{~km} \mathrm{~s}^{-1}$.

34 This threshold halo mass is similar to the one used in Section 4.2 where we tested effects of dynamical friction using the $z=0$ snapshot.
} 
We compute the galaxy surface densities around the resultant subsamples by projecting along the entire $z$-axis in the simulation. We subtract the background surface density mimicking the procedure used to compute the surface densities in the data. The error bars on these surface densities are obtained using 125 jackknife regions in the simulation box. The resulting surface density profiles and their error bars are shown in the top left panel of Figure 10. The measurements in the simulations are carried out using the same binning scheme as employed for the actual data analysis.

We fit these measured surface density profiles using the projection of the three-dimensional DK14 density profile with exactly the same priors as detailed in Section 2. The $68 \%$ and $95 \%$ confidence intervals on the fit are shown using the shaded regions in the top left panel of Figure 10. The inferred slope of the surface density profiles and the location of the steepest slope in two dimensions are shown in the top right panel.

The points in the bottom left panel correspond to the threedimensional density profiles of these subhalos as measured from the simulation directly. The solid lines are used to indicate the three-dimensional subhalo density profiles as predicted by the best fit to the subhalo surface density profiles in the upper panels. Finally, in the bottom right panel we compare the inferred and measured logarithmic slopes of the threedimensional density profiles. This test shows that our analysis methods can reproduce the location of the splashback radius by fitting the two-dimensional density profiles of subhalos in the simulations.

\section{APPENDIX B \\ ABUNDANCE MATCHING CONSTRAINTS ON THE SUBHALOS HOSTING PHOTOMETRIC GALAXIES}

We use the surface number density distribution of photometric galaxies around our cluster subsamples to detect the splashback radius. In this appendix, we use a simple subhalo abundance matching technique to determine the properties of subhalos hosting the photometric galaxies we use in this paper. The left-hand panel of Figure 11 shows the cumulative abundance of galaxies, based on the Schechter function fit to the ${ }^{0.1} i$-band luminosity function for SDSS galaxies obtained by Blanton et al. (2003). ${ }^{35}$

We $k$-correct the absolute magnitude limits of the photometric galaxies we use, and correct them for the luminosity evolution of galaxies ( $e$-correction) from $z=0.24$, the median redshift of our cluster subsamples, to $z=0.1$. We approximate the $k$-correction as

$$
{ }^{0.1} k(z)=-2.5 \log _{10}\left[\frac{z+1.3}{1.1(0.1+1.3)}\right],
$$

found by fitting the $k$-correction as a function of redshift using the SDSS main sample of spectroscopic galaxies. We have ignored the residual color-dependent scatter in this relation. These $k+e$ corrected magnitude limits for our photometric subsamples are shown with vertical dashed lines, while the horizontal dashed lines show their cumulative abundances. We do not assume any scatter between $V_{\text {peak }}$ and magnitude, to obtain a limit on the maximum effect that dynamical friction can have, and match these abundances directly to those of subhalos as a function of $V_{\text {peak }}$. The result of this simple subhalo

\footnotetext{
${ }^{35}$ The notation ${ }^{0.1} i$ stands for magnitude in the $i$-band $k$-corrected to $z=0.1$.
}

abundance matching exercise is shown in the right-hand panel of Figure 11.

The abundance matching implies that the subhalos hosting our fiducial subsample of photometric galaxies approximately correspond to subhalos with $V_{\text {peak }}>135 \mathrm{~km} \mathrm{~s}^{-1}$, while the brighter subsamples correspond to subhalos with $V_{\text {peak }}>175 \mathrm{~km} \mathrm{~s}^{-1}$ and $V_{\text {peak }}>280 \mathrm{~km} \mathrm{~s}^{-1}$, respectively. In Section 4.2, these abundance matching constraints are used to explore how dynamical friction is expected to affect the location of the splashback radius for our subsamples.

\section{APPENDIX C ADDITIONAL INVESTIGATIONS REGARDING PROJECTION AND HALO ORIENTATION EFFECTS}

Galaxy cluster halos are expected to be triaxial, and if the major axes of the clusters in one of our subsamples were preferentially aligned along the line of sight, we could overestimate the halo mass of that particular subsample (see e.g., Oguri et al. 2005). We have performed the following tests to evaluate such possibility. Any attempts to explain the halo assembly bias signal based on projection or orientation effects should also satisfy these observational constraints.

We have utilized the spectroscopic coverage of the SDSSDR12 LOWZ galaxy sample to investigate the velocity distributions of such galaxies around our cluster subsamples. If one of our subsamples is heavily affected by projection effects, then the velocity distributions around the two subsamples should reveal differences. The redMaPPer cluster catalog contains spectroscopic redshifts for the 3037 (2830) most probable central galaxies in the high- (low-) $c_{\text {gal }}$ subsamples. We show the $P(\Delta V)$ distribution of all spectroscopic galaxies within a projected radius of $R_{200 \mathrm{~m}}$ of the cluster center in the left-hand panel of Figure 12. We find very little evidence, if any, of a difference between the two distributions.

The photometric major axis of central galaxies has been reported to have a preferential alignment with the major axis of the dark matter halo, albeit with a reasonable scatter (see, e.g., Evans \& Bridle 2009; Oguri et al. 2010, 2012). If one of our subsamples (the high- $c_{\mathrm{gal}}$ subsample) had major axes which were preferentially aligned with the line of sight, we expect the brightest cluster galaxy ellipticities of that sample to be rounder than the other. In the middle panel of Figure 12, we show the axis ratio distributions of the light distributions in various SDSS bands, as reported by the SDSS photometric pipeline, of the most probable central galaxies in our two subsamples. These axis ratios were computed by fitting a de Vaucouleurs profile to the two-dimensional image of each object in each band (Stoughton et al. 2002). We do not see any strong evidence for a difference in the axis ratios of the most probable central galaxies between the two cluster subsamples.

The satellite galaxy distribution is also expected to have a preferential alignment with the major axis of the dark matter halo (see e.g., Zentner et al. 2005; Kang et al. 2007). We compute the second moment of the projected satellite galaxy distribution around our cluster subsamples using the membership probabilities of all member galaxies as reported in the redMaPPer catalog. We use these second moments to compute the major and minor axes, and their axis ratios, around the most probable central galaxies. In the right-hand panel of Figure 12, we show the axis ratio distributions of the member galaxy distributions. Again we see very little difference between the two cluster subsamples. 

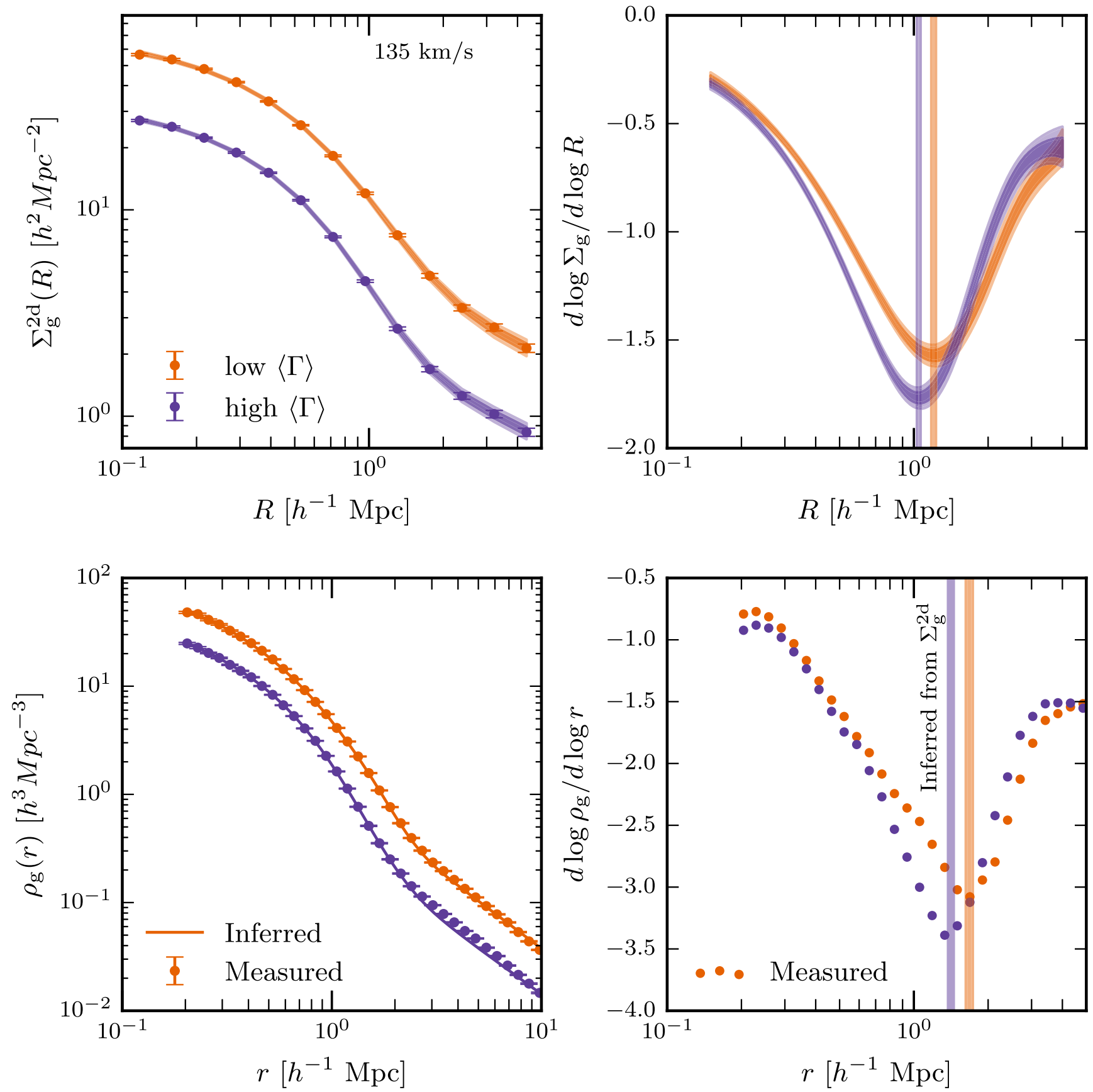

Figure 10. Tests of our methodology to determine the splashback radius of clusters. Top left: the subhalo surface density profiles around high and low accretion rate clusters in the MDPL2 simulation at $z=0$ are shown using purple and orange symbols with error bars, respectively. The data points for the low accretion rate clusters are shifted by a factor of 2.5 for clarity. The $68 \%$ and $95 \%$ confidence intervals based on fitting these data using the DK14 model are shown using shaded regions. Top right: the logarithmic derivative of the surface density profiles around the mock cluster subsamples as inferred from a model fit to the data. The vertical shaded bands correspond to the location of the steepening in two dimensions. Bottom left: the comparison between the three-dimensional subhalo density profiles around our mock clusters, as directly measured and as inferred from the fits to the surface density profiles from the top left panel. Bottom right: Comparison between the logarithmic derivative of the three-dimensional subhalo density profiles around our mock clusters, as directly measured and as inferred from the fits to the surface density profiles from the top left panel. The $68 \%$ constraints on the location of the splashback radius are shown using the shaded vertical bands. Our analytical methods can recover the location of the splashback radius in three dimensions.

\section{APPENDIX D \\ MAGNIFICATION DUE TO CLUSTERS}

The background density of photometric galaxies behind clusters is expected to be different from the background density around random points due to magnification effects by galaxy clusters. We consider a simple estimate of this effect based on the weak lensing mass estimate of our clusters. The magnification changes the number counts in two ways: first, fainter galaxies can now enter our sample after being magnified by the cluster, and second, the background galaxies occupy a smaller solid angle behind the cluster than in the absence of magnification.

For simplicity, we assume that all our clusters are located at the median redshift of our subsample. We assume that background galaxies follow the luminosity function presented 

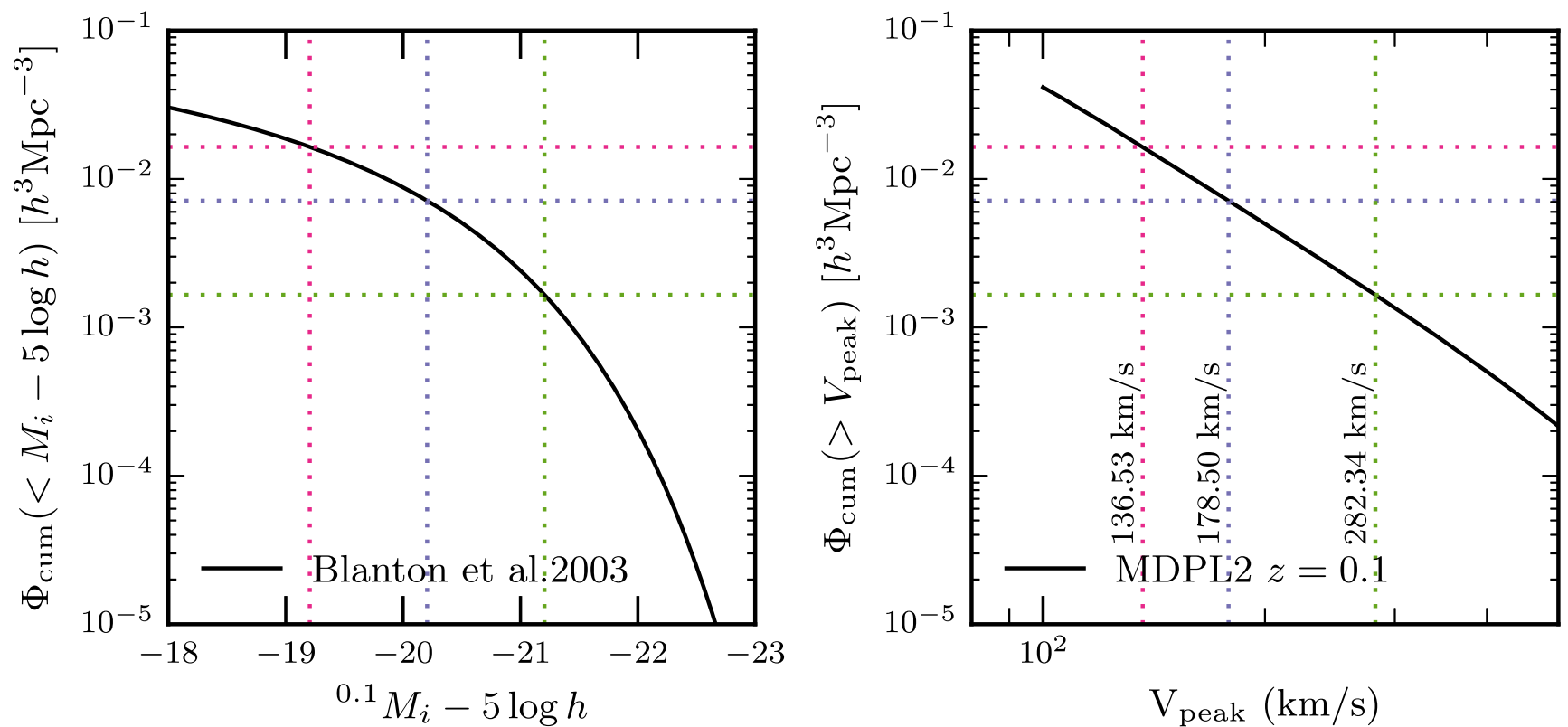

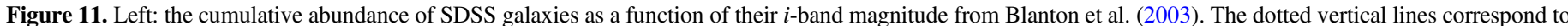

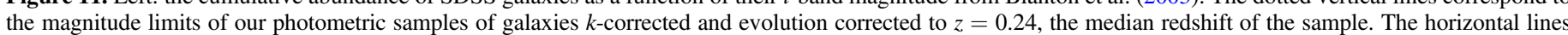

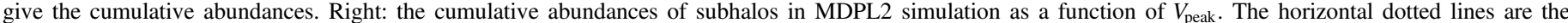

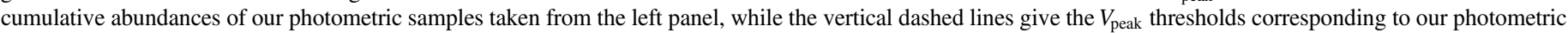
subsamples. These $V_{\text {peak }}$ thresholds are used to test dynamical friction effects on the location of the splashback radius (see Figure 7).
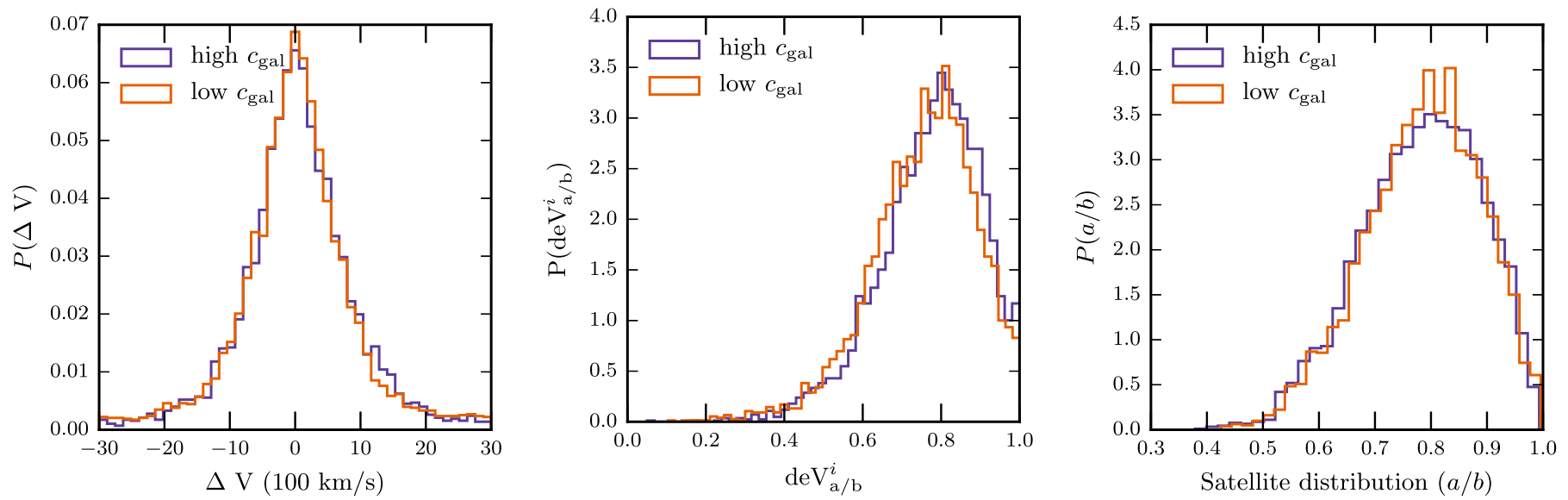

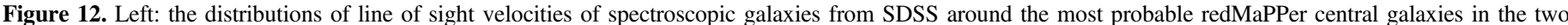

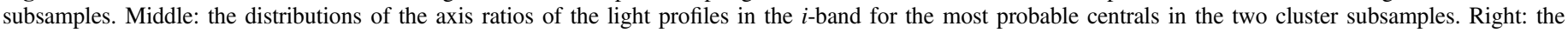

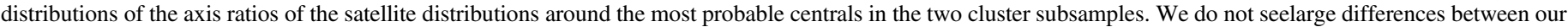
two cluster subsamples for any of these statistics.

by Blanton et al. (2003). The number of galaxies that will be observed at a projected distance $R$ from the cluster is then given by

$$
N\left(R \mid z_{\mathrm{l}}, z_{\mathrm{s}}\right)=\int d z_{\mathrm{s}} \frac{d V}{d z_{\mathrm{s}}} \frac{1}{\mu\left(R \mid z_{\mathrm{l}}, z_{\mathrm{s}}\right)} \Phi\left(M<M_{\max }^{\prime} \mid z_{\mathrm{s}}\right)
$$

where $\mu$ denotes the magnification due to the cluster at a projected distance $R$, and

$$
\begin{aligned}
M_{\max }^{\prime}= & M_{\max }-5.0 \log _{10}\left(D_{l}\left[z_{\mathrm{s}}\right] / D_{l}\left[z_{\mathrm{l}}\right]\right) \\
& +2.5 \log _{10} \mu\left(R \mid z_{\mathrm{l}}, z_{\mathrm{s}}\right) .
\end{aligned}
$$

Here $M_{\max }$ corresponds to the maximum absolute magnitude for the galaxies that end up in our subsample. In this paper, we have considered three different thresholds, $M_{\max }-5 \log h=-19.43$,
-20.43 , and -21.43 . The above equation accounts for the fact that we have assumed the redshift of the photometric galaxies to be equal to that of the lens redshift while converting their apparent magnitudes to absolute magnitudes. We additionally $k+e$ correct $M_{\max }$ to $z=0.1$ as the luminosity function we use is based on such $k+e$ corrected magnitudes.

In Figure 13, we plot the ratio between the number counts obtained by using Equation (5) with $\mu$ obtained for a halo of mass $M_{200 \mathrm{~m}}$ that matches the weak lensing estimate for our cluster subsamples. We see that due to the magnification of our clusters, we should be underestimating our background at smaller radii by $5 \%-10 \%$, depending upon the photometric sample under consideration, although the corrections to the backgrounds near the splashback radii are about $1 \%$. To check for a systematic effect arising from magnification effects, we multiply the number 


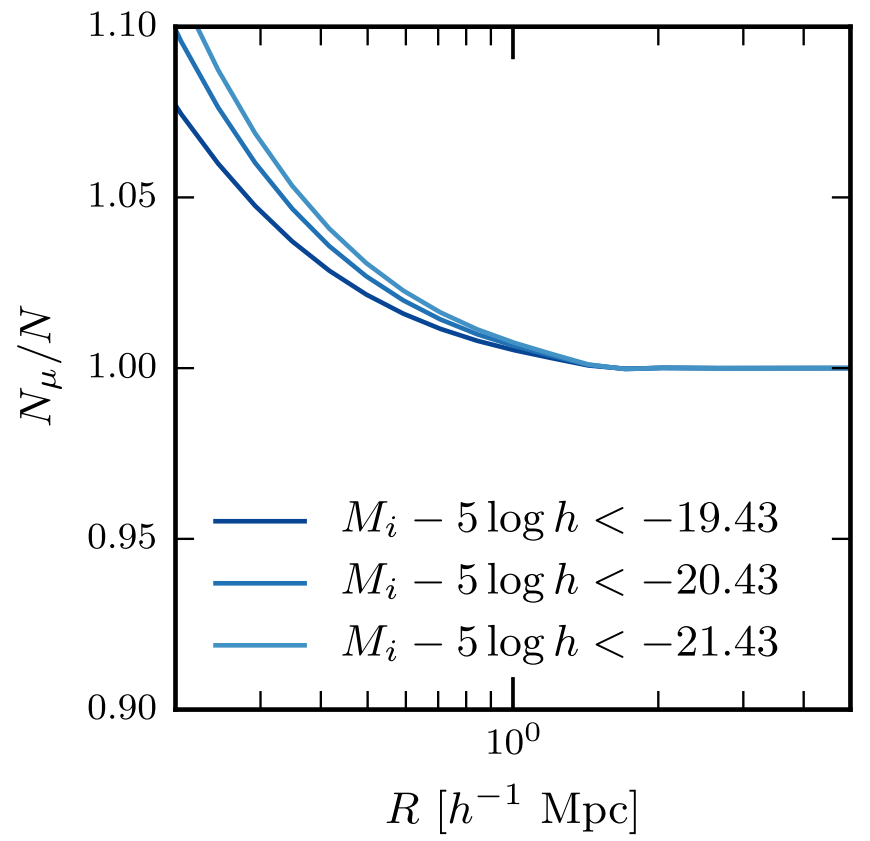

Figure 13. Magnification effects on the background estimation: the ratio of the number counts of background galaxies behind a cluster compared to that around random points, for the three different photometric samples we use in this paper. On the scales of interest for the splashback radius, the corrections to the background estimate are at the percent level. We have checked that the splashback radius estimates are not affected by such small changes to the background.

counts around random points by this ratio before subtracting them from the number of galaxies around our cluster subsamples. Note that this is a conservative estimate of the effect, because this factor should only be applied to the background galaxies, whereas the number of galaxies around random points also includes foreground galaxies. We have confirmed that the location of the splashback radius is insensitive to such a change. If anything, it is expected to be lowered by such a change, given that we are underestimating the background.

\section{REFERENCES}

Adhikari, S., Dalal, N., \& Chamberlain, R. T. 2014, JCAP, 11, 19 Adhikari, S., Dalal, N., \& Clampitt, J. 2016, arXiv:1605.06688 Aihara, H., Allende, P., Carlos, A., et al. 2011, ApJS, 193, 29

Bardeen, J. M., Bond, J. R., Kaiser, N., \& Szalay, A. S. 1986, ApJ, 304, 15 Becker, M. R., \& Kravtsov, A. V. 2011, ApJ, 740, 25

Behroozi, P. S., Wechsler, R. H., \& Wu, H.-Y. 2013, ApJ, 762, 109

Bertschinger, E. 1985, ApJS, 58, 39

Blanton, M. R., Hogg, D. W., Bahcall, N. A., et al. 2003, ApJ, 592, 819

Dalal, N., White, M., Bond, J. R., \& Shirokov, A. 2008, ApJ, 687, 12

Diemer, B., \& Kravtsov, A. V. 2014, ApJ, 789, 1

Elbert, O. D., Bullock, J. S., Garrison-Kimmel, S., et al. 2015, MNRAS, 453, 29

Evans, A. K. D., \& Bridle, S. 2009, ApJ, 695, 1446

Fillmore, J. A., \& Goldreich, P. 1984, ApJ, 281, 1

Foreman-Mackey, D., Hogg, D. W., Lang, D., \& Goodman, J. 2013, PASP, 125,306

Gao, L., Navarro, J. F., Cole, S., et al. 2008, MNRAS, 387, 536
Gao, L., Springel, V., \& White, S. D. M. 2005, MNRAS, 363, L66

Gao, L., \& White, S. D. M. 2007, MNRAS, 377, L5

Gnedin, O. Y., \& Ostriker, J. P. 2001, ApJ, 561, 61

Goodman, J., \& Weare, J. 2010, Commun. Appl. Math. Comput. Sci., 5, 65

Harvey, D., Massey, R., Kitching, T., Taylor, A., \& Tittley, E. 2015, Sci, 347, 1462

Hearin, A. P., Watson, D. F., \& van den Bosch, F. C. 2015, MNRAS, 452, 1958

Hikage, C., Mandelbaum, R., Takada, M., \& Spergel, D. N. 2013, MNRAS, 435,2345

Jiang, F., \& van den Bosch, F. C. 2016, MNRAS, 458, 2848

Jiang, L., Helly, J. C., Cole, S., \& Frenk, C. S. 2014, MNRAS, 440, 2115

Kahlhoefer, F., Schmidt-Hoberg, K., Frandsen, M. T., \& Sarkar, S. 2014, MNRAS, 437, 2865

Kahlhoefer, F., Schmidt-Hoberg, K., Kummer, J., \& Sarkar, S. 2015, MNRAS, 452, L54

Kaiser, N. 1984, ApJL, 284, L9

Kang, X., van den Bosch, F. C., Yang, X., et al. 2007, MNRAS, 378, 1531

Klypin, A., Yepes, G., Gottlöber, S., Prada, F., \& Heß, S. 2016, MNRAS, 457, 4340

Kravtsov, A. V., \& Borgani, S. 2012, ARA\&A, 50, 353

Li, Y., Mo, H. J., \& Gao, L. 2008, MNRAS, 389, 1419

Lin, Y.-T., Mandelbaum, R., Huang, Y.-H., et al. 2016, ApJ, 819, 119

Mandelbaum, R., Hirata, C. M., Seljak, U., et al. 2005, MNRAS, 361, 1287

Miyatake, H., More, S., Takada, M., et al. 2016, PhRvL, 116, 041301

Mo, H. J., \& White, S. D. M. 1996, MNRAS, 282, 347

More, S. 2013, ApJL, 777, L26

More, S., Diemer, B., \& Kravtsov, A. V. 2015, ApJ, 810, 36

More, S. 2016, Astrophysics Source Code Library, ascl:1601.012

Navarro, J. F., Frenk, C. S., \& White, S. D. M. 1996, ApJ, 462, 563

Niikura, H., Takada, M., Okabe, N., Martino, R., \& Takahashi, R. 2015, PASJ, 67, 103

Oguri, M., Bayliss, M. B., Dahle, H., et al. 2012, MNRAS, 420, 3213

Oguri, M., \& Hamana, T. 2011, MNRAS, 414, 1851

Oguri, M., Takada, M., Okabe, N., \& Smith, G. P. 2010, MNRAS, 405, 2215

Oguri, M., Takada, M., Umetsu, K., \& Broadhurst, T. 2005, ApJ, 632, 841

Patej, A., \& Loeb, A. 2015, arXiv:1509.07506

Randall, S. W., Markevitch, M., Clowe, D., Gonzalez, A. H., \& Bradač, M. 2008, ApJ, 679, 1173

Rines, K., Geller, M. J., Diaferio, A., \& Kurtz, M. J. 2013, ApJ, 767, 15

Rozo, E., Rykoff, E. S., Becker, M., Reddick, R. M., \& Wechsler, R. H. 2015, MNRAS, 453, 38

Rykoff, E. S., Rozo, E., Busha, M. T., et al. 2014, ApJ, 785, 104

Rykoff, E. S., Rozo, E., Hollowood, D., et al. 2016, ApJS, 224, 1

Schlegel, D. J., Finkbeiner, D. P., \& Davis, M. 1998, ApJ, 500, 525

Sheth, R. K., Mo, H. J., \& Tormen, G. 2001, MNRAS, 323, 1

Sheth, R. K., \& Tormen, G. 2004, MNRAS, 350, 1385

Simet, M., McClintock, T., Mandelbaum, R., et al. 2016, arXiv:1603.06953

Skibba, R. A., van den Bosch, F. C., Yang, X., et al. 2011, MNRAS, 410, 417

Spergel, D. N., \& Steinhardt, P. J. 2000, PhRvL, 84, 3760

Stoughton, C., Lupton, R. H., Bernardi, M., et al. 2002, AJ, 123, 485

Tinker, J. L., George, M. R., Leauthaud, A., et al. 2012, ApJL, 755, L5

Tinker, J. L., Robertson, B. E., Kravtsov, A. V., et al. 2010, ApJ, 724, 878

Tully, R. B. 2015, AJ, 149, 54

Umetsu, K., Broadhurst, T., Zitrin, A., Medezinski, E., \& Hsu, L.-Y. 2011, ApJ, 729, 127

Villaescusa-Navarro, F., Bird, S., Peña-Garay, C., \& Viel, M. 2013, JCAP, 3, 019

Villaescusa-Navarro, F., \& Dalal, N. 2011, JCAP, 3, 024

Vogelsberger, M., Mohayaee, R., \& White, S. D. M. 2011, MNRAS, 414, 3044

Wechsler, R. H., Zentner, A. R., Bullock, J. S., Kravtsov, A. V., \& Allgood, B. 2006, ApJ, 652, 71

Yang, X., Mo, H. J., \& van den Bosch, F. C. 2006, ApJL, 638, L55

Zentner, A. R., Kravtsov, A. V., Gnedin, O. Y., \& Klypin, A. A. 2005, ApJ, 629,219 